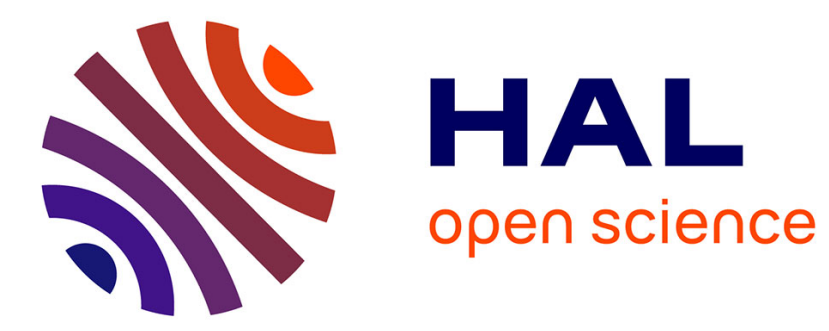

\title{
Measuring inconsistency and deriving priorities from fuzzy pairwise comparison matrices using the knowledge-based consistency index
}

Sylvain Kubler, William Derigent, Alexandre Voisin, Jérérmy Robert, Yves Le Traon, Enrique Herrera Viedma

\section{To cite this version:}

Sylvain Kubler, William Derigent, Alexandre Voisin, Jérérmy Robert, Yves Le Traon, et al.. Measuring inconsistency and deriving priorities from fuzzy pairwise comparison matrices using the knowledge-based consistency index. Knowledge-Based Systems, 2018, 162, pp.147-160. 10.1016/j.knosys.2018.09.015 . hal-01883339

\section{HAL Id: hal-01883339 \\ https://hal.univ-lorraine.fr/hal-01883339}

Submitted on 28 Sep 2018

HAL is a multi-disciplinary open access archive for the deposit and dissemination of scientific research documents, whether they are published or not. The documents may come from teaching and research institutions in France or abroad, or from public or private research centers.
L'archive ouverte pluridisciplinaire HAL, est destinée au dépôt et à la diffusion de documents scientifiques de niveau recherche, publiés ou non, émanant des établissements d'enseignement et de recherche français ou étrangers, des laboratoires publics ou privés. 


\title{
Measuring Inconsistency and Deriving Priorities from Fuzzy Pairwise Comparison Matrices using the Knowledge-based Consistency Index
}

\author{
Sylvain Kubler ${ }^{\mathrm{a}, \mathrm{b}, *}$, William Derigent ${ }^{\mathrm{a}, \mathrm{b}}$, Alexandre Voisina ${ }^{\mathrm{a}, \mathrm{b}}$, Jérérmy Robert $^{\mathrm{c}}$, Yves Le Traon ${ }^{\mathrm{c}}$, Enrique Herrera \\ Viedma $^{\mathrm{d}}$ \\ ${ }^{a}$ Université de Lorraine, CRAN, UMR 7039, Campus Sciences, BP 70239, VandÅuvre-lès-Nancy F-54506, France \\ ${ }^{b}$ CNRS, CRAN, UMR 7039, France \\ ${ }^{c}$ University of Luxembourg, Interdisciplinary Centre for Security, Reliability $\mathcal{E}$ Trust \\ 4 rue Alphonse Weicker L-2721 Luxembourg \\ ${ }^{d}$ University of Granada, Departamento de Ciencias de la Computación e Inteligencia Artificial \\ Daniel Saucedo Aranda, s/n 18071 Granada España
}

\begin{abstract}
The fuzzy analytic hierarchy process (AHP) is a widely applied multiple-criteria decision-making (MCDM) technique, making it possible to tackle vagueness and uncertainty arising from decision makers, especially in a pairwise comparison process. Indeed, as the human brain reasons with uncertain rather than precise information, pairwise comparisons may involve some degree of inconsistency, which must be correctly managed to guarantee a coherent result/ranking. Several consistency indexes for fuzzy pairwise comparison matrices (FPCMs) have been proposed in the literature. However, some scholars argue that most of these fail to be axiomatically grounded, which may lead to misleading results. To overcome this lack of an axiomatically grounded index, a new index is proposed in this paper, referred to as the knowledge-based consistency index $(\mathrm{KCI})$. A comparative study of the proposed index with an existing one is carried out, and the results show that KCI contributes to substantially reducing the computation time. In addition, the different fuzzy weights derived from the initial FPCM (for KCI computation purposes) can also be employed to find a crisp set of weights that corresponds to an optimal solution to the MCDM problem according to the decision maker's viewpoint and expertise.
\end{abstract}

Keywords: Multiple criteria decision-making, Analytic hierarchy process (AHP), Fuzzy logic, Consistency, Decision analysis

\section{Introduction}

According to some specialists in human judgments, it has been proven that the human brain can only consider a limited amount of information at one time [1], making it unreliable for making decisions regarding complex problems (i.e., with multiple and conflicting parameters). Hence, multiple-criteria decision-making (MCDM) methods have been introduced to help decision makers overcome this issue. MCDM methods can be classified into two categories [2]: multi-attribute decision-making (MADM) and multi-objective decision-making (MODM). Unlike MODM, MADM techniques involve considerable human participation. One of the most widely employed MADM techniques is the analytic hierarchy process (AHP), initially introduced by Saaty [3]. Its main strengths lie in its objective and logical ranking system, and its flexibility to be jointly used with other techniques such as fuzzy logic, neural networks and SWOT (strengths, weaknesses, opportunities, threats) analysis [4, 5]. However, this technique requires the decision makers to express their knowledge in a consistent manner. Indeed, in AHP knowledge assessment is performed by carrying out pairwise comparisons between a set of items (criteria or alternatives). More specifically, decision

\footnotetext{
${ }^{*}$ Corresponding author

Email addresses: s.kubler@univ-lorraine.fr (Sylvain Kubler), william.derigent@univ-lorraine.fr (William Derigent), alexandre.voisin@univ-lorraine.fr (Alexandre Voisin), jeremy.robert@uni.lu (Jérérmy Robert), yves.letraon@uni.lu (Yves Le Traon), viedma@decsai.ugr.es (Enrique Herrera Viedma)
} 
makers must specify by "how many more times item i is preferred to item $j$ ”. Because human beings typically reason using "local" information (i.e., one pairwise comparison at the same time) rather than with global information (i.e., taking into account the whole set of pairwise comparisons at a time), such a process may introduce some degree of inconsistency $[6,7]$. To overcome this problem, Saaty introduced a consistency ratio (CR) that aims to measure the degree of inconsistency for a given pairwise comparison matrix. When this ratio exceeds $10 \%$, a judgment often needs reexamination. According to [8], consistency indexes can be classified into two categories: (i) "intra" expert consistency, which focuses on a single decision maker/matrix $[9,10]$, and (ii) "inter" expert consistency, which focuses on inconsistency analyses resulting from a group of decision makers [11,8].

Fuzzy logic has been introduced in AHP, in an approach more commonly known as fuzzy AHP (FAHP), as a way to cope with uncertainty and vagueness arising in knowledge assessment. This has found significant applications in recent years [5, 12]. Unlike the classical set theory, fuzzy logic enables the gradual assessment of the membership of elements in relation to a set [13]. The first FAHP method was introduced by van Laarhoven and Pedrycz [14] in 1983. Since then, many other methods have been introduced, as reviewed in a recent state-of-the-art survey of FAHP applications [5].

Similarly to AHP, the consistency also has to be quantified in FAHP. In 1985, Buckley [15] proposed a first consistency index for fuzzy pairwise comparison matrices (FPCMs). Several similar indexes have since been introduced, including the fuzzy logarithmic least squares consistency [14], the feasible region consistency [16], the fuzzy preference-programming consistency [17], the additive consistency [6,18, 19], and the geometric consistency [20, 21]. Despite their various advantages and disadvantages, several theoretical calculation problems and questions have been raised concerning the introduction of fuzzy sets in AHP, especially with regard to the axiomatic foundation of the approach [22, 23]. Dubois [22] argues that fuzzy sets have often been incorporated in to existing methods, such as PROMETHEE and ELECTRE, without clear benefits. He also adds that fuzzy sets in AHP must be considered, first and foremost, at the "axiomatic" level, not simply at the technical one. Looking more closely at the reasons behind such criticisms, one may find that a major problem lies in the difficulty of successfully satisfying the transitivity and reciprocal axioms $[24,25,26]$.

Existing consistency indexes are more thoroughly reviewed and discussed in Section 2, with discussions spanning from their evolution over time to their pros and cons. Following this literature review, a new index, referred to as "Knowledge-based Consistency Index" (KCI), is introduced in Section 3. This is evaluated and compared with a known consistency index in section 4. Finally, conclusions are provided in section 5 . Let us note that a preliminary version of this research work was presented to the IEEE International Conference on Fuzzy Systems (FUZZ-IEEE) in Naples, July 2017 [27]. The present article extends this work through (i) a more in-depth literature review of existing consistency indexes, (ii) a new section detailing the mathematical formulation of crisp FPCM's weight derivation, and (iii) a more complete evaluation and comparison study considering a wider range of FPCMs (237 in this article against 48 in the conference version).

\section{Consistency Indexes in Fuzzy AHP: An Overview}

The AHP method starts by structuring the problem in a hierarchal manner (goal, criteria and alternative levels), followed by a pairwise comparison process between such items. These different steps can be formalized as follows:

1. An $n \times n$ consistent matrix $A$ (denoted by $A^{n \times n}$ ) is used to model the pairwise relative preferences of $n$ items. Each $a_{i j}$ coefficient is supposed to reflect the factor by which the $i^{\text {th }}$ item is preferred to the $j^{\text {th }}$.

2. A consistent matrix must fulfill both the reciprocal and transitivity axioms, which can respectively be expressed as: (i) $a_{i j}=a_{i k} \cdot a_{k j} \forall i, j, k, i \neq k$ and (ii) $a_{j i}=\frac{1}{a_{i j}} \forall i, j$.

3. The largest eigenvalue of the matrix is equal to $n$, and a corresponding eigenvector $w=\left(w_{1}, w_{2}, \ldots, w_{n}\right)$ (with $\left.\forall i, j, a_{i j}=\frac{w_{i}}{w_{j}}\right)$ can be found.

AHP is among the most popular techniques for dealing with MCDM problems. However, some scholars, such as Dubois [22], argue that: "asking for precise values $a_{i j}$ is debatable, because these coefficients are arguably imprecisely known by experts.”. Furthermore, approaches have thus been introduced to handle such imprecisions, the principle of which consists of extending the computational approach proposed by Saaty with fuzzy intervals [5]. A fuzzy pairwise 


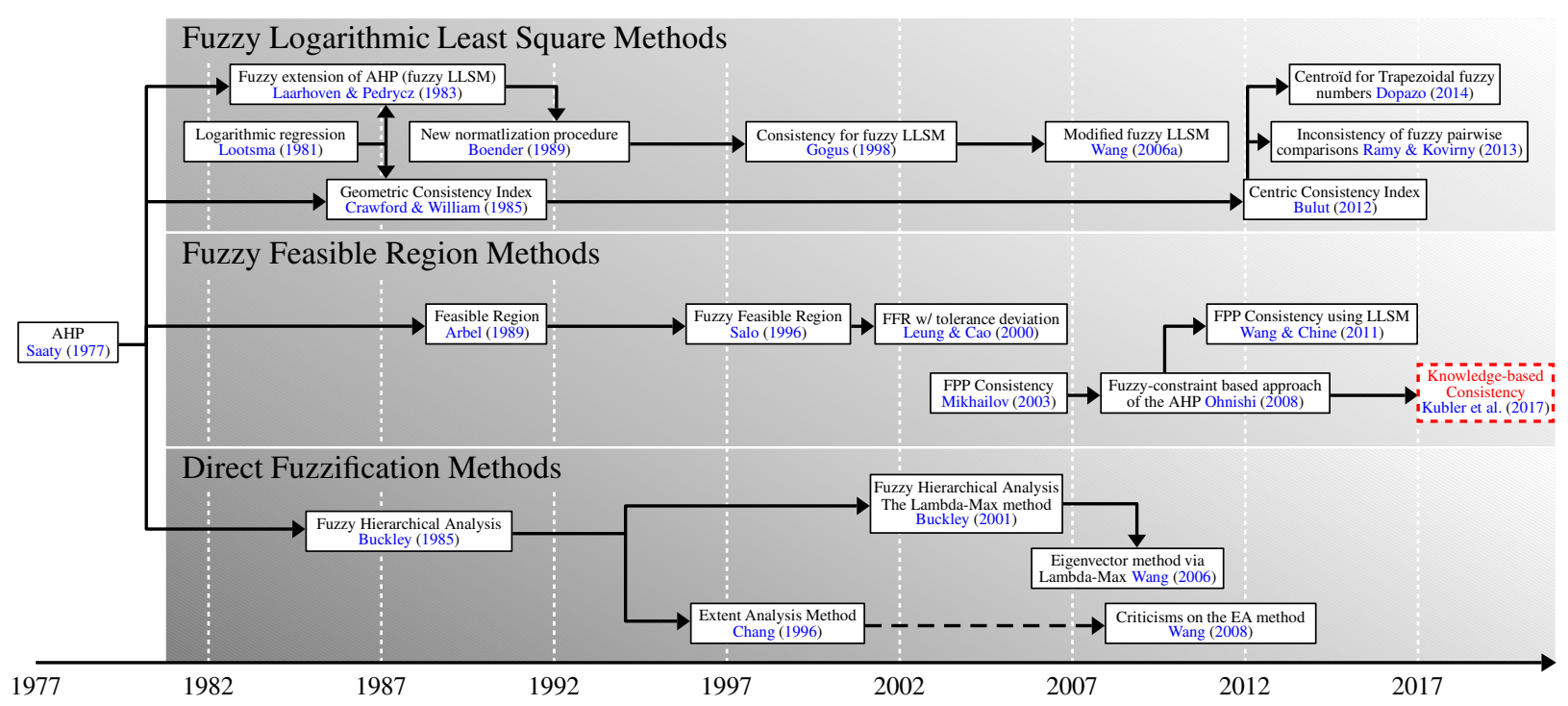

Figure 1: Overview of existing consistency indexes since the introduction of the analytic hierarchy process

comparison number, denoted by $\tilde{a}_{i j}$ in (1), is supposed to reflect the expert preference - when comparing items $i$ and $j$ - with some level of imprecision. Since the introduction of AHP [28], various methods have been proposed to manage inconsistency in FPCMs. These methods can be classified into three categories: (i) fuzzy logarithmic least square (FFLS), (ii) direct fuzzification (DF), and (iii) fuzzy feasible region (FFR). Figure 1 presents an overview of the evolution of consistency indexes since the introduction of AHP (1977). Each of these categories and associated papers are further discussed in Sections 2.1 through 2.4.

$$
\tilde{A}=\left[\tilde{a}_{i j}\right]=\begin{array}{cccc}
1 & 2 & \ldots & n \\
2 & \vdots \\
n & {\left[\begin{array}{cccc}
\tilde{a}_{11} & \tilde{a}_{12} & \ldots & \tilde{a}_{1 n} \\
\tilde{a}_{21} & \tilde{a}_{22} & \ldots & \tilde{a}_{2 n} \\
\vdots & \vdots & \vdots & \vdots \\
\tilde{a}_{n 1} & \tilde{a}_{n 2} & \ldots & \tilde{a}_{n n}
\end{array}\right]}
\end{array}
$$

\subsection{Fuzzy logarithmic least square (FFLS) indexes}

van Laarhoven and Pedrycz [14] extended the work of Lootsma [29] on logarithmic least square methods (LLSM) to FPCMs, leading to the so-called fuzzy LLSM. These methods produce fuzzy weights from FPCMs using logarithmic regression. Let $\tilde{A}$ be a group fuzzy matrix expressed as in (B.1) ${ }^{1}$, where $\tilde{a}_{i j k}=\left(l_{i j k}, m_{i j k}, u_{i j k}\right)$ are triangular fuzzy judgments. The authors state that there should exist a normalized triangular fuzzy weight vector $\tilde{W}=\left(\tilde{w}_{1}, \ldots, \tilde{w}_{n}\right)=\left(\left(w_{1}^{L}, w_{1}^{M}, w_{1}^{U}\right), \ldots,\left(w_{n}^{L}, w_{n}^{M}, w_{n}^{U}\right)\right)$ close to $\tilde{A}$, according to (B.2). To determine the fuzzy weight vector $\tilde{W}$, the authors proposed an FFLS model as given in (B.3). Boender et al. [30] showed that the procedure proposed by [14] to normalize the fuzzy weights was inappropriate, owing to the fact that it could lead to a non-optimal solution. Gogus and Boucher [31] also pointed out this limitation, adding that it can lead to irrational fuzzy weights (i.e., $w_{i}^{L}>w_{i}^{U}$ ). To tackle this problem, the authors introduced the notion of strong transitivity in FPCMs, which represents a direct extension of Saaty's transitivity axiom. An FPCM $\tilde{A}^{n \times n}$ where $\tilde{a}_{i j}=\left(a_{i j l}, a_{i j m}, a_{i j u}\right)$ is strongly transitive if $a_{i j m} \cdot a_{j k m}=a_{i k m}$ and $\sqrt{a_{i j u} \cdot a_{i j l}} \cdot \sqrt{a_{j k u} \cdot a_{j k l}}=\sqrt{a_{i k u} \cdot a_{i k l}} ; \forall i, j, k$. This condition ensures that the results of fuzzy LLSM lead to coherent fuzzy weights. Wang et al. [32] proposed a modified version of fuzzy LLSM by both introducing a new normalization procedure and handling inconsistent FPCMs. Other existing fuzzy LLSM consistency measures are based on extending the geometric consistency index [20], which was later completed by [33].

\footnotetext{
${ }^{1}$ See Tables B.3 and B.4 given in Appendix B.
} 
Bulut et al. [34] introduced the centric consistency index (CCI) for triangular FPCMs, as formalized in (B.4), where $\tilde{A}^{n \times n}$ is a triangular FPCM for which $\tilde{w}=\left[\left(w_{L_{1}}, w_{M_{1}}, w_{U_{1}}\right), \ldots,\left(w_{L_{n}}, w_{M_{n}}, w_{U_{n}}\right)\right]$ is the derived priority vector obtained by use of the row geometric mean method. Dopazo et al. [35] further extended CCI to trapezoidal fuzzy matrices using a centroid defuzzification approach [36], as shown in (B.5). Ramík and Korviny [21] proposed an additional method of solving the FAHP problem following a two-step process: (i) an optimal weight vector $\tilde{W}$ (with the solution being unique and having the minimal spread) is generated by using the geometric mean method as shown in (B.6) to (B.8), which is then (ii) employed to compute a consistent FPCM $\tilde{X}=\frac{\tilde{w}_{i}}{\tilde{w}_{j}}$. More specifically, the metric given in (B.9) is adopted to compute the distance $N I_{n}^{\sigma}(\tilde{A})$ between $\tilde{A}$ and $\tilde{X}$, where $\gamma_{n}^{\sigma}$ is a normalized constant that measures the inconsistency of $\tilde{A}$. Therefore, $\tilde{A}$ is said to be $F$-consistent if $\gamma_{n}^{\sigma}(\tilde{A})=0$, and is called $F$-inconsistent otherwise. In a more recent article, Ramík and Korviny [37] proposed another index called $F I(\tilde{A})$, which measures the inconsistency of $\tilde{A}$, as shown in (B.10).

\subsection{Direct fuzzification (DF) methods}

The second category includes approached that attempt to directly "fuzzify" AHP by applying the extension principle to the original method. Buckley [15] introduced the fuzzy hierarchical analysis, and proposed a fuzzy extension of Saaty's consistency definition. In subsequent work, the $\lambda$-max method has also been directly extended by Csutora and Buckley [38], and improved in [39]. Buckley advocates that an FPCM respecting $\tilde{a}_{i k} \odot \tilde{a}_{k j} \approx \tilde{a}_{i j}$ is compliant with Saaty's consistency definition. A final method of determining the fuzzy weights of the fuzzy priority vector was introduced by Chang [40], and employs an extension of the crisp simplified computation of the different weights, as well as the degree of possibility for the defuzzification of the fuzzy priority vector.

\subsection{Fuzzy feasible region (FFR) methods}

FFR methods are based on the work of Arbel [41], which was further extended by Salo [16]. In this approach, an $\tilde{A}^{n \times n}$ FPCM is considered as consistent if there exists a set of crisp relative weights whose ratios are within the limits implies by the different elements of $\tilde{A}$. The feasible region corresponds to the set of weight vectors respecting the different fuzzy constraints expressed via $\tilde{A}$. Using $\alpha$-cuts, Salo formalized the feasible region $S_{\alpha}$ as shown in (B.11). The existence of a value of $\alpha>0$, for which $S_{\alpha}$ is not empty, ensures that there is at least one weight vector that solves the MCDM problem. In subsequent works, Leung and Cao [42] introduced the notion of tolerance deviation, to take into account cases in which $\tilde{A}$ is not consistent, therefore proposed a modified expression of the feasible region. The authors considered that an FPCM is consistent if $S_{1}$ (i.e., for $\alpha=1$ ) is not empty. This notion is debatable, as will be further discussed in Section 2.4.

Mikhailov [43] defined a fuzzy preference programming method to find crisp priorities from FPCMs, represented as normal convex fuzzy sets. Using $\alpha$-cuts, each $\tilde{a}_{i j}$ can be represented as a sequence of sets denoted by $a_{i j}\left(\alpha_{l}\right), l=$ $\{1, \ldots, L\}$, where $0=\alpha_{1}<\ldots<\alpha_{L}=1$. $\tilde{A}$ can be converted into a series of $L$ interval sets $F_{l}=\left\{a_{i j}\left(\alpha_{l}\right)\right\} \mid l=$ $\{1, \ldots, L\}$. Mikhailov's main idea is to find a crisp weight vector that satisfies each interval set, to the best possible extent, and aggregate the result to obtain the final crisp values of a weight vector for the entire FPCM, as expressed in (B.12) (where $\tilde{\leq}$ denotes the "best possible extent" statement). Considering $m$ as the number of pairwise comparison judgments expressed by the decision maker $\left(m \leq \frac{n(n-1)}{2}\right)$, the author shows that (B.12) is equivalent to a set of $2 m$ fuzzy constraints that can be expressed as a matrix, as given in (B.13). The $k^{\text {th }}$ row of (B.13), denoted by $R_{k} \cdot w \tilde{\leq} 0 \mid k=$ $\{1, \ldots, 2 m\}$ represents a fuzzy linear constraint. This can be characterized by a linear membership function, as given in (B.14) (where $d_{k}$ is a tolerance parameter representing the admissible interval for approximate satisfaction of the crisp inequality $R_{k} \cdot w \leq 0$ ). The fuzzy feasible area $\tilde{P}$ is a fuzzy set, described by the membership function given in (B.15). The maximized solution is then the crisp vector $w^{*}$ corresponding to the maximum membership degree of $\tilde{P}$, as given in (B.16). As stated in [44], this is a typical max-min fuzzy linear problem, which can be transformed into a conventional linear program and be solved using optimization methods (where $\mu_{\tilde{P}}\left(w^{*}\right)$ measures the degree of satisfaction of the fuzzy constraints). Mikhailov argues that this is a natural indicator of the consistency of the decision maker's judgments, since $\mu_{\tilde{P}}\left(w^{*}\right)=1$ for consistent judgments, and $\leq 1$ otherwise.

Similarly, Ohnishi et al. [45] considered two matrices: (i) an FPCM denoted by $\tilde{A}$, for which each fuzzy element $\tilde{a}_{i j}$ is viewed as a flexible constraint; and (ii) a crisp matrix $A=a_{i j}$, which is consistent according to Saaty's definition (i.e., $a_{i j}=a_{i k} \cdot a_{k j} \forall i, j, k, i \neq k$ ). This implies that there exists an $n$-tuple of weights, denoted by $w^{*}=\left\{w_{1}, \ldots, w_{n}\right\}$, whose sum is equal to 1 , such that $\forall i, j, a_{i j}=\frac{w_{i}}{w_{j}}$. Given this, Ohnishi defines the consistency of the fuzzy constraints 
of $\tilde{A}$ as the degree to which an AHP-consistent matrix $A$ exists and satisfies the fuzzy constraint expressed in $\tilde{A}$. The authors define the consistency degree as in (B.17), where its best fitting weight patterns can be determined using (B.18). This problem constitutes a max-min problem that can be converted to an optimization problem using $\alpha$-cuts. One interesting point concerning Ohnishi's method is that the solution is unique, and by nature, compliant with Saaty's consistency definition.

Wang and Chin [17] proposed the logarithmic fuzzy preference programming methodology, combining the work of [43] with logarithmic regression. The authors argue that this method solves some issues of the previous methods, such as negative membership degree and multiple optimal solutions, and more. The authors take the logarithm of the FPCM using the approximate equation given in (B.19). The logarithm of a fuzzy element $\tilde{a}_{i j}$ can still be seen as an approximate triangular fuzzy number, whose membership function can be defined as in (B.20), where $\mu_{i j}\left(\ln \left(w_{i} / w_{j}\right)\right)$ is the membership degree of $\ln \left(w_{i} / w_{j}\right)$. Similarly to [45] and [43], the authors seek a crisp priority vector $w^{*}$ to maximize the minimum membership degree $\mu\left(w^{*}\right)=\min \left\{\mu_{i j}\left(\ln \left(w_{i} / w_{j}\right)\right)\right\}$, which can be considered as a consistency index varying from 0 (strongly inconsistent) to 1 (fully consistent).

\subsection{Discussion of methods and consistency measures}

As discussed in Sections 2.1-2.2, fuzzifying AHP has led to three main categories of methods, each with having specific properties:

- FFLS aims at deriving fuzzy weights from FPCMs using optimization techniques (the obtained weights can be unrealistic depending on the applied technique, especially if FPCMs do not satisfy strong transitivity);

- DF extends part of the original AHP, and its objective is to produce fuzzy priority vectors. Some of the proposed techniques do not require the use of optimization methods;

- FFR takes fuzzy matrices as flexible constraints (or can also sometimes consider fuzzy operators), and its objective is to compute (using optimization methods) a crisp optimal priority vector that is unique.

Despite the fact that the above techniques have been the subject of criticisms in the literature, they remain widely employed in FAHP applications, as shown through a recent state-of-the-art survey of FAHP applications [5]. Among other criticisms, Saaty and Tran [46] argued that it is erroneous to fuzzify AHP because Saaty's scale is intrinsically fuzzy. Wang et al. [24] advocate that the fuzzy extent analysis suffers from theoretical pitfalls, leading to incorrect results. The attempts of $[38,39]$ lead to eigenvalues that are not even reciprocal. The natural extension of a simple crisp equation $a \cdot x=b$ (let alone an eigenvalue problem) does not necessarily result in a fuzzy equation of the type $\tilde{a} \cdot \tilde{x}=\tilde{b}$. Dubois [22] argued that DF methods raise some concerns: (i) Replacing a consistent preference matrix by a fuzzy-valued preference one leads to a loss of the properties of the former; (ii) it is very difficult to rigorously define fuzzy eigenvalues of vectors; and (iii) considering an interval-matrix defined by $\alpha$-cut intervals, denoted by $\tilde{a}_{i j}^{\alpha}=\left[\underline{a}_{i j} ; \bar{a}_{i j}\right]$, leads to other issues (e.g., the boundary matrices are no longer reciprocal). Dubois also noted that Fuzzy LLSM approaches, and particularly those developed in [21,37], do not reveal much about the scalar distance between the underlying precise ones. Zhü [26] also claimed that methods to compute fuzzy weight vectors from FPCMs lead to several violations of both the AHP axioms and fuzzy logic. In fact, it seems that the philosophy underpinning these two categories (fuzzy LLSM and DF) is not correctly founded. However, Dubois considers that viewing fuzzy pairwise preference data as an imprecise knowledge is an interesting approach, adding that a constraintbased view of FAHP is promising. This is why FFR methods require special attention. The different FFR methods proposed so far take into account the consistency aspect, as the notion of a fuzzy feasible region refers to the fact that there exists an AHP-compliant crisp matrix that is compatible with the fuzzy constraints. The formalization proposed by Salo [16] in (B.11) does not measure the level of consistency of the crisp matrix. Mikhailov [43] considered the inconsistency of a crisp matrix as linked to the number of elements of the matrix outside of the limits of the corresponding FPCM. The related value of inconsistency is computed as the adjustment required in order not to violate the crisp inequalities. This work raises two questions: (i) What is the consequence of this adjustment on the final solution? and (ii) To what extent can the decision maker augment this parameter and consider the solution priority vector as acceptable? In our opinion, accepting to breach the limits of the flexible constraints is not a valid approach, because it adds "fuzziness on fuzzy sets." That said, the approach developed by Ohnishi et al. [45] appears to us to be mathematically well-founded: The obtained crisp vector respects the fuzzy constraints given by the FPCM, 


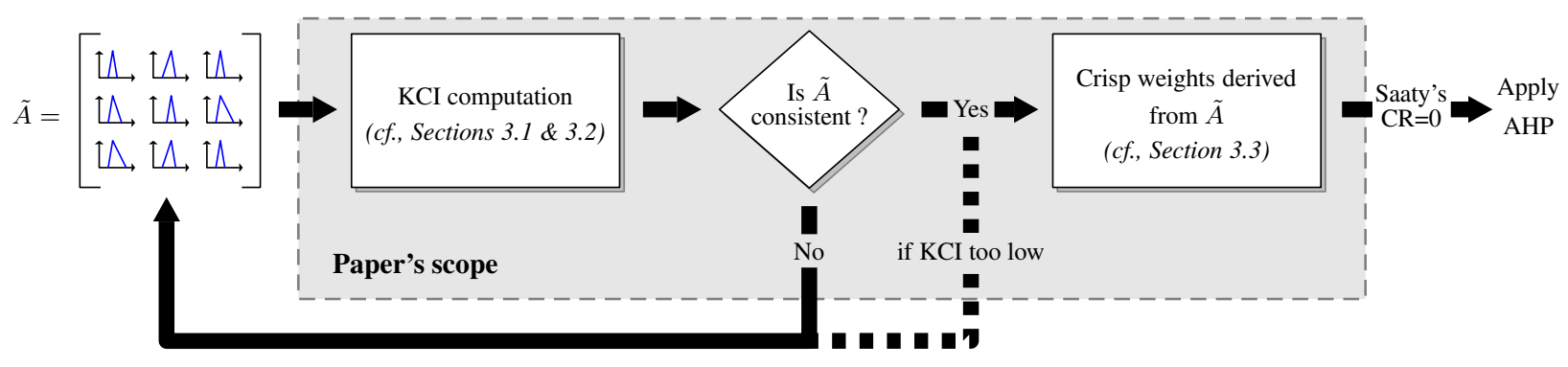

Figure 2: Knowledge-based consistency index (KCI) workflow: from the (in)consistency check to the derivation of crisp weights

while also satisfying Saaty's consistency definition. Viewing the FPCM as representing knowledge about preference relations is the research direction followed in our study. Nonetheless, it may be interesting to ensure that the fuzzy constraints expressed in the FPCM are compatible with each other. To do so, in the next section we propose a new consistency index that expresses the compatibility of the different fuzzy constraints.

\section{Knowledge-based Consistency Index}

The literature review from the previous section shows that most of today's consistency indexes fail to be suitably "axiomatically" grounded, which may lead to misleading results. To overcome this problem, a new index, KCI, is introduced in this section. Figure 2 provides an insight into the workflow set up for both computing KCI and deriving crisp weights from the input FPCM (denoted by $\tilde{A}$ in Figure 2). It should be noted that the scope of this paper is limited to the verification of whether $\tilde{A}$ is (or not) consistent according to KCI's definition, and if so to the derivation of a consistent crisp matrix according to Saaty's definition that satisfies the fuzzy constraints expressed in $\tilde{A}$ (i.e., leading to $\mathrm{CR}=0$ ). That is, this research does not aim to provide decision makers with recommendations on (i) how to tune $\tilde{A}$ to make it consistent ( $c f$., the arrow denoted by "No" in Figure 2); or (ii) the extent to which a KCI score is (or not) sufficient to deem $\tilde{A}$ as "consistent" ( $c f$., the arrow denoted by "KCI too low"). That said, we aim to study such questions in future research, as will be discussed in section 5.2.

As highlighted in Figure 2, the formalization of KCI and the underlying mathematical proof are respectively presented in Sections 3.1 and 3.2, while Section 3.3 details how a crisp consistent pairwise comparison matrix and its normalized eigenvector can be derived based on KCI.

\subsection{KCI formalisation}

The term "knowledge" will be used as a reference to all possible values that a variable $x$ can have from an expert viewpoint, meaning that a given value is associated with a preference degree. Using fuzzy sets and FPCMs to model this knowledge is one possible approach, where the decision maker's knowledge relates to two types of knowledge:

- Direct expert knowledge when comparing item $i$ to $j$ (i.e., $\tilde{a}_{i j}$ );

- Indirect expert knowledge resulting from the transitivity axiom $\tilde{a}_{i k} \otimes \tilde{a}_{k j}$.

However, as emphasized by Dubois [22], the crisp transitivity axiom is not appropriate when dealing with human knowledge, because such knowledge is granular rather than crisp. A strict equality (=) between fuzzy sets indicates that all of $\tilde{a}_{i j}$ values would belong to $\tilde{a}_{i k} \otimes \tilde{a}_{k j}$, and vice-versa. This implies that the crisp Saaty's transitivity axiom cannot be directly applied as it is not feasible in practice to comply with such an axiom when using fuzzy sets. A weaker condition would consist of checking whether items of "knowledge" are consistent. That is, whether there exists a common element $\tilde{a}_{i j} \cap\left(\tilde{a}_{i k} \otimes \tilde{a}_{k j}\right)$. This leads to the following expression when considering the whole FPCM:

$$
\left(\cap\left(\tilde{a}_{i k} \otimes \tilde{a}_{k j}\right) \cap \tilde{a}_{i j}\right) \neq \varnothing
$$


Although the above condition allows for checking the extent to which the direct and indirect expert knowledge is compatible (i.e., $\tilde{a}_{i j}$ ), it does not provide any indication about the consistency degree of $\tilde{A}$, as it only returns a binary result ( $\tilde{A}$ is or is not consistent). To overcome this lack of indicator, in this paper we introduce a new consistency index: the Knowledge-based Consistency Index $\left(\alpha_{\mathrm{KCI}}\right)$. This index is derived from (2), except that the inclusion operator is used rather than the equal or non-disjunction operators. This can be formulated as in (3), where the left term relates to the indirect knowledge and the fuzzy inclusion operator relates to the matching degree between the indirect and direct expert knowledge.

$$
\cap\left(\tilde{a}_{i k} \otimes \tilde{a}_{k j}\right) \supseteq \tilde{a}_{i j}
$$

The inclusion operator $\supseteq$ is introduced in order to quantify the consistency level of $\tilde{A}$, as given in (4). This means that the function maps the element $\tilde{a} \tilde{\supseteq} \tilde{b}$ to the element "sup $\sup _{x \in \mathcal{R}}\left(\min \left(\mu_{\tilde{a}}(x), \mu_{\tilde{b}}(x)\right)\right)$ ".

$$
\begin{aligned}
\tilde{\supseteq}: \tilde{\mathfrak{R}} \times \tilde{\mathfrak{R}} & \longrightarrow[0,1] \\
\tilde{a} \tilde{\supseteq} \tilde{b} & \mapsto \sup _{x \in \mathfrak{R}}\left(\min \left(\mu_{\tilde{a}}(x), \mu_{\tilde{b}}(x)\right)\right)
\end{aligned}
$$

Finally, $\alpha_{\mathrm{KCI}}$ can be defined as the minimum satisfaction degree that can be attained via the entire FPCM, which in some way reflects the extent to which the transitivity axiom can be satisfied $(c f .,(3))$. This can be formalized as in (5):

$$
\alpha_{\mathrm{KCI}}=\min _{i, j \in \mathcal{N} \mid i<j}\left[\sup \left[\left(\underset{k \in \mathcal{N}-\{i, j\}}{\bigcap}\left(\tilde{a}_{i k} \otimes \tilde{a}_{k j}\right)\right) \tilde{\supseteq} \tilde{a}_{i j}\right]\right]
$$

If $0<\alpha_{\mathrm{KCI}}<1$, then it can be stated that $\tilde{A}$ is "partly" consistent with the $\alpha$ level, because a minimal compatibility between the direct and indirect expert knowledge can be reached, no matter how small the compatibility is. If $\alpha_{\mathrm{KCI}}=1$, then the FPCM is said to be "perfectly" consistent, which means that the direct expert knowledge is fully included in the indirect knowledge. In contrast, if $\alpha_{\mathrm{KCI}}=0$ then $\tilde{A}$ is said to be inconsistent, because for one of the $\frac{n(n-1)}{2}$ pairwise comparisons, the direct expert knowledge is not included in the indirect knowledge. Algorithm 1 synthesizes all the steps necessary to compute $\alpha_{\mathrm{KCI}}$, and further to check whether $\tilde{A}$ is or not consistent.

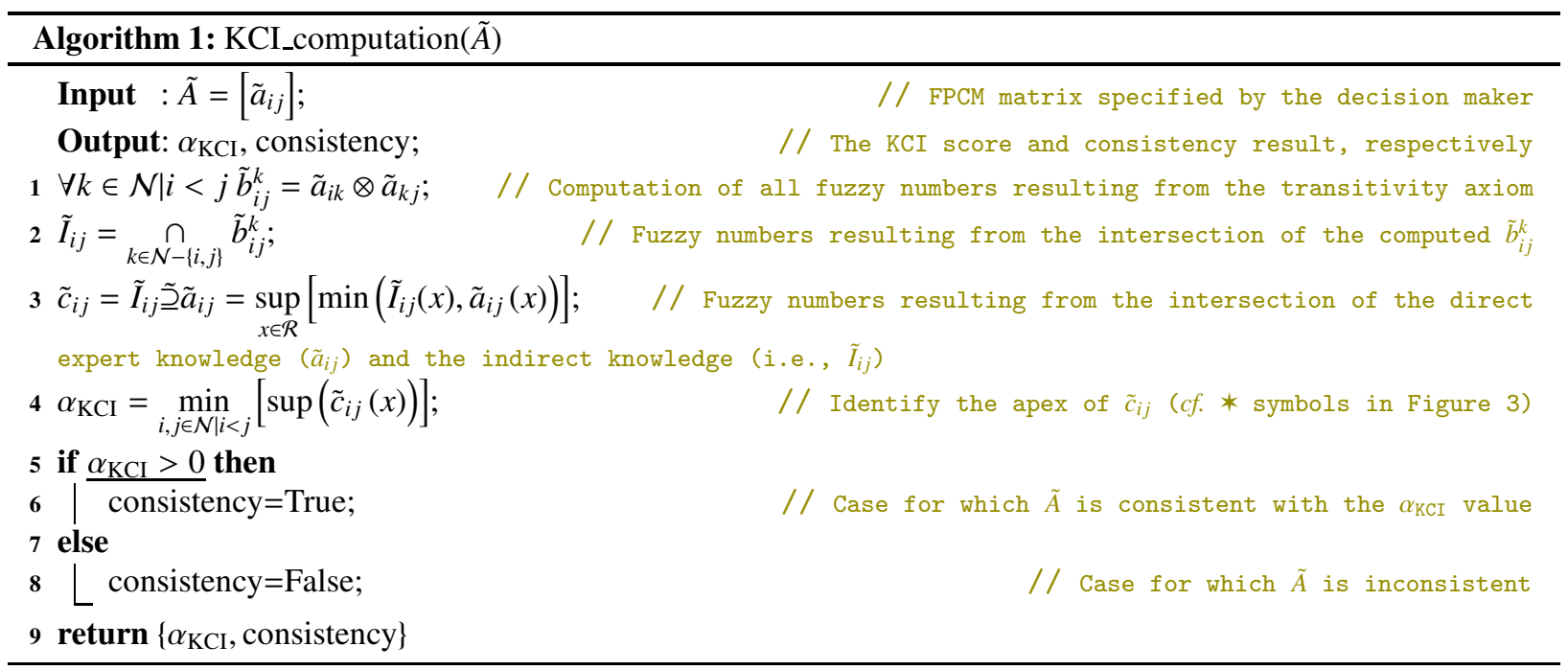

Deriving a crisp consistent matrix from a fuzzy one is often the subject of debate, owing to the lack of mathematical rigour. Given this, Section 3.2 details a mathematical proof of how $\alpha_{\mathrm{KCI}}$ is linked to the consistency of a crisp matrix. 


\subsection{Relation between $\alpha_{K C I}$ and crisp matrix consistency}

Let $\tilde{A}$ be a triangular FPCM, and $A^{\alpha}=\left[\tilde{a}_{i j}^{\alpha}\right]$ its $\alpha$-cut ${ }^{2}$ matrix, also called alpha-matrix, which consists of the $\alpha$-cuts of each element of $\tilde{A}$.

Definition 1 (alpha-matrix consistency). $A^{\alpha}$ is consistent if and only if (6) is satisfied. All $w_{i}$ that do respect this inequality are together called the "feasible region" [16].

$$
\exists w_{i}, w_{j} \in \mathbb{R}^{+}, \underline{a}_{i j}^{\alpha} \leq \frac{w_{i}}{w_{j}} \leq \bar{a}_{i j}^{\alpha}, \forall \tilde{a}_{i, j}
$$

The above definition is similar to that given by [48], as it leads to a vector $W=\left(w_{1}, w_{2}, \ldots, w_{n}\right)$ that can be derived from $A^{\alpha}$ whose values $a_{i j}$ are always between $\underline{a}_{i j}^{\alpha}$ and $\bar{a}_{i j}^{\alpha}$ :

Theorem 1 (alpha-matrix consistency check). $A^{\alpha}$ is a consistent matrix if and only if:

$$
\max _{k}\left(\underline{a}_{i j}^{\alpha} ; \underline{a}_{i k}^{\alpha} \times \underline{a}_{k j}^{\alpha}\right) \leq \min _{k}\left(\bar{a}_{i j}^{\alpha} ; \bar{a}_{i k}^{\alpha} \times \bar{a}_{k j}^{\alpha}\right), \forall i, j, k
$$

Proof. Following Definition 1, it can be stated that if $A^{\alpha}$ is a consistent matrix, then it implies that the feasible region is not empty, and that no conflict exists between the following inequality constraints exists:

$$
\begin{array}{r}
\underline{a}_{i k}^{\alpha} \leq w_{i} / w_{k} \leq \bar{a}_{i k}^{\alpha}, i, k=1, \ldots, n \\
\underline{a}_{k j}^{\alpha} \leq w_{k} / w_{j} \leq \bar{a}_{k j}^{\alpha}, k, j=1, \ldots, n \\
\underline{a}_{i j}^{\alpha} \leq w_{i} / w_{j} \leq \bar{a}_{i j}^{\alpha}, i, j=1, \ldots, n
\end{array}
$$

Multiplying (8) by (9) gives rise to the following inequality:

$$
\underline{a}_{i k}^{\alpha} \times \underline{a}_{k j}^{\alpha} \leq w_{i} / w_{j} \leq \bar{a}_{i k}^{\alpha} \times \bar{a}_{k j}^{\alpha}, i, j, k=1, \ldots, n
$$

Furthermore, (10) and (11) imply the following inequality:

$$
\max \left(\underline{a}_{i j}^{\alpha} ; \underline{a}_{i k}^{\alpha} \times \underline{a}_{k j}^{\alpha}\right) \leq w_{i} / w_{j} \leq \min \left(\bar{a}_{i j}^{\alpha} ; \bar{a}_{i k}^{\alpha} \times \bar{a}_{k j}^{\alpha}\right)
$$

Because (12) is valid for any $k=\{1, \ldots, n\}$, it can be noted that $\max _{k}\left(\underline{a}_{i j}^{\alpha} ; \underline{a}_{i k}^{\alpha} \times \underline{a}_{k j}^{\alpha}\right) \leq \min _{k}\left(\bar{a}_{i j}^{\alpha} ; \bar{a}_{i k}^{\alpha} \times \bar{a}_{k j}^{\alpha}\right)$ is valid for all $i, j, k=\{1, \ldots, n\}$.

Theorem 2 (relation between knowledge and alpha-matrix consistency). If Ã is a knowledge-based consistent matrix for the $\alpha$-level (measured using $\alpha_{K C I}$ ), then $A^{\alpha}$ can be said to be consistent.

Proof. Based on (2) and (3), $\tilde{A}$ can be said to be a knowledge-based consistent matrix if and only if the following equation holds for all $\tilde{a}_{i j}$ :

$$
\begin{aligned}
\tilde{c}_{i j} & \neq \emptyset \\
\text { with } \tilde{c}_{i j} & =\left(\left(\underset{k \in \mathcal{N}-\{i, j\}}{\cap}\left(\tilde{a}_{i k} \otimes \tilde{a}_{k j}\right)\right) \cap \tilde{a}_{i j}\right)
\end{aligned}
$$

Applying alpha-cuts to (14) leads to the following:

$$
\begin{array}{r}
\tilde{c}_{i j}=\tilde{a}_{i j} \cap\left(\tilde{a}_{i k} \otimes \tilde{a}_{k j}\right) \\
\Rightarrow c_{i j}^{\alpha}=a_{i j}^{\alpha} \cap\left(a_{i k}^{\alpha} \times a_{k j}^{\alpha}\right) \\
\Rightarrow\left[\underline{c}_{i j}^{\alpha} ; \bar{c}_{i j}^{\alpha}\right]=\left[\underline{a}_{i j}^{\alpha} ; \bar{a}_{i j}^{\alpha}\right] \cap\left(\left[\underline{a}_{i k}^{\alpha} ; \bar{a}_{i k}^{\alpha}\right] \times\left[{ }^{\alpha} \underline{a}_{k j}^{\alpha} ; \bar{a}_{k j}^{\alpha}\right]\right) \\
\Rightarrow\left[\underline{c}_{i j}^{\alpha} ; \bar{c}_{i j}^{\alpha}\right]=\left[\underline{a}_{i j}^{\alpha} ; \bar{a}_{i j}^{\alpha}\right] \cap\left[\underline{a}_{i k}^{\alpha} \times \underline{a}_{k j}^{\alpha} ; \bar{a}_{i k}^{\alpha} \times \bar{a}_{k j}^{\alpha}\right] \\
\Rightarrow\left[\underline{c}_{i j}^{\alpha} ; \bar{c}_{i j}^{\alpha}\right]=\left[\max \left(\underline{a}_{i j}^{\alpha} ; \underline{a}_{i k}^{\alpha} \times \underline{a}_{k j}^{\alpha}\right) ; \min \left(\bar{a}_{i j}^{\alpha} ; \bar{a}_{i k}^{\alpha} \times \bar{a}_{k j}^{\alpha}\right)\right]
\end{array}
$$

(19) is equivalent to Theorem 1, meaning that when knowledge-based consistency is satisfied at the $\alpha$-level for $\tilde{A}$, its corresponding alpha-matrix $\left(A^{\alpha}\right)$ is also consistent. Let us add that if $\alpha_{\mathrm{KCI}}>0$, then it is always possible to derive/find a consistent crisp matrix from $\tilde{A}$. Such a weight derivation process is detailed in Section 3.3.

\footnotetext{
${ }^{2}$ An $\alpha$-cut level of $\tilde{A}$ corresponds to the crisp set $A^{\alpha}$ such as $A^{\alpha}=\left\{x \in \mathbb{R} \mid \mu_{\tilde{A}}(x) \geq \alpha\right\}$ with $\left.\left.\alpha \in\right] 0,1\right]$. By definition, $A^{0}=\left\{x \in \mathbb{R} \mid \mu_{\tilde{A}}(x) \neq 0\right\}$ $\mu_{\tilde{A}}(x) \neq 0$ for $A^{0}$.
} 


\subsection{PCM derivation from FPCM}

In order to obtain the normalized eigenvector of the FPCM $\tilde{A}$, a fuzzy matrix denoted by $\tilde{C}^{\mathrm{KCI}}$ is firstly derived from $\tilde{A}$, the elements of which are $\tilde{c}_{i j}$, as defined in (14). Next, let $C^{\mathrm{KCI}}$ denote the crisp consistent matrix to be found, the corresponding elements $c_{i j}$ of which are derived from $\tilde{c}_{i j}$. Each element $\tilde{c}_{i j}$ synthetizes the decision maker's knowledge issued from the direct knowledge, and the indirect knowledge of the $i^{\text {th }}$ row and $j^{\text {th }}$ column is obtained from the transitivity axiom ( $c f$., Section 2). Because the reciprocal axiom is considered to be satisfied by $\tilde{A}$, a similar consideration is followed for $\tilde{C}^{\mathrm{KCI}}$, thus leading us to only consider the upper-half elements of $\tilde{C}^{\mathrm{KCI}}\left(\right.$ i.e., $\left.\tilde{c}_{i j} \mid i<j\right)$.

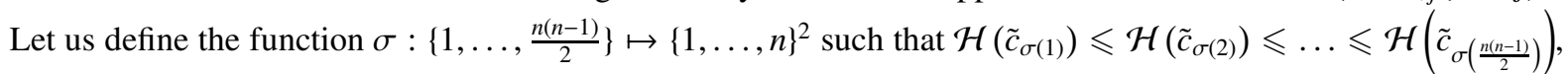
where $\mathcal{H}$ is the height of the fuzzy set. That is, the largest membership degree of the fuzzy set $\mathcal{H}(\tilde{A})=\sup _{x}\left(\mu_{\tilde{A}}(x)\right)$. $\sigma$ orders the elements of $\tilde{C}^{\mathrm{KCI}}$ in an increasing order of their height, and thus $\tilde{c}_{\sigma(1)}$ is the element that provides the KCI value. Let us first consider $\tilde{c}_{\sigma(1)}$, because it has a lower height, and by definition a lower consistency. The obvious choice for the value is that at which the membership function $\tilde{c}_{\sigma(1)}$ attains its maximum, which is denoted by $c_{\sigma(1)}$. Since in our study we only consider triangular FPCMs, $\mathcal{H}\left(\tilde{c}_{\sigma(1)}\right)$ is obtained as a unique value for the support. According to Theorem 2, we know that $\forall l \in\{2, \ldots, n(n-1) / 2\}$ the values in the $\alpha$-cuts $\tilde{c}_{\sigma(l)}^{\mathcal{H}\left(\tilde{c}_{\sigma(1)}\right)}$ are consistent with $c_{\sigma(1)}$, meaning that there exists a crisp solution for $c_{\sigma(l)} \forall l \neq 1$. Then, in a similar manner, we consider the value for $c_{\sigma(2)}$ where the membership function $\tilde{c}_{\sigma(2)}$ attains its maximum. According to Theorem 2, we also know that $\forall l \in\{3, \ldots, n(n-1) / 2\}$, the values in the $\alpha$-cuts $\tilde{c}_{\sigma(l)}^{\mathcal{H}\left(\tilde{c}_{\sigma(2)}\right)}$ are consistent with $c_{\sigma(2)}$ as well as $c_{\sigma(1)}$. Similarly, $c_{\sigma(3)}$ is defined as the value at which the membership function $\tilde{c}_{\sigma(3)}$ attains its maximum. Overall, for all $\tilde{c}_{i, j}$ we thus consider $c_{i, j} \mid i<j$ as the value at which the membership function $\tilde{c}_{i, j}$ attains its maximum. Finally, the elements of $C^{\mathrm{KCI}}$ are being defined as in (20):

$$
c_{i, j}=x \mid \max _{x}\left(\tilde{c}_{i, j}(x)\right)
$$

\section{Implementation and Evaluation of KCI}

Section 4.1 provides a practical implementation of the computational stages underlying $\alpha_{\mathrm{KCI}}$. Section 4.2 presents an in-depth analysis of the computational behavior of $\alpha_{\mathrm{KCI}}$, based on which the algorithm parameters are determined and set up. Section 4.3 presents a comparison study between our index $\left(\alpha_{\mathrm{KCI}}\right)$ and that proposed by Ohnishi et al. [45] (denoted by $\alpha_{\mathrm{Ohn}}$ ). Section 4.4 analyses the impact of using the highly criticized - but still widely employed - extent analysis method of Chang on the consistency results.

Note that the following studies were carried out using MATLAB (R2014b) under an Intel Pentium Core i7-2677m environment (CPU: $1.80 \mathrm{GHz}$, memory: 4GB).

\subsection{Implementation of $K C I$}

In this section, a triangular FPCM is selected from [49], where an expert carries out pairwise comparisons between four emergency response capacities, denoted by $\mathrm{C} 1, \mathrm{C} 2, \mathrm{C} 3$, and $\mathrm{C} 4 \mathrm{in} \tilde{A}$ :

$$
\begin{aligned}
& \begin{array}{llll}
\mathrm{C} 1 & \mathrm{C} 2 & \mathrm{C} 3 & \mathrm{C} 4
\end{array} \\
& \tilde{A}=\begin{array}{l}
\mathrm{C} 2 \\
\mathrm{C} 3 \\
\mathrm{C} 4
\end{array}\left[\begin{array}{cccc}
(1,1,1) & \left(\frac{3}{2}, 2, \frac{5}{2}\right) & \left(\frac{2}{3}, 1,2\right) & \left(1, \frac{3}{2}, 2\right) \\
\tilde{a}_{21}^{-1} & (1,1,1) & \left(\frac{2}{3}, 1,2\right) & \left(\frac{1}{2}, \frac{2}{3}, 1\right) \\
\tilde{a}_{31}^{-1} & \tilde{a}_{32}^{-1} & (1,1,1) & \left(\frac{1}{2}, \frac{2}{3}, 1\right) \\
\tilde{a}_{41}^{-1} & \tilde{a}_{42}^{-1} & \tilde{a}_{43}^{-1} & (1,1,1)
\end{array}\right]
\end{aligned}
$$

Figure 3 provides a graphical overview of $\tilde{A}$, where the membership functions in blue/solid relate to the different $\tilde{a}_{i j}$ elements, and the dashed/red ones to the indirect expert knowledge, i.e., $\left(\tilde{a}_{i j} \otimes \tilde{a}_{j k}\right)$. It can be observed that more than one red fuzzy set results from this operation, because $\operatorname{dim}(\tilde{A})>3$. Let us detail the calculation regarding $\tilde{a}_{12}$ for 


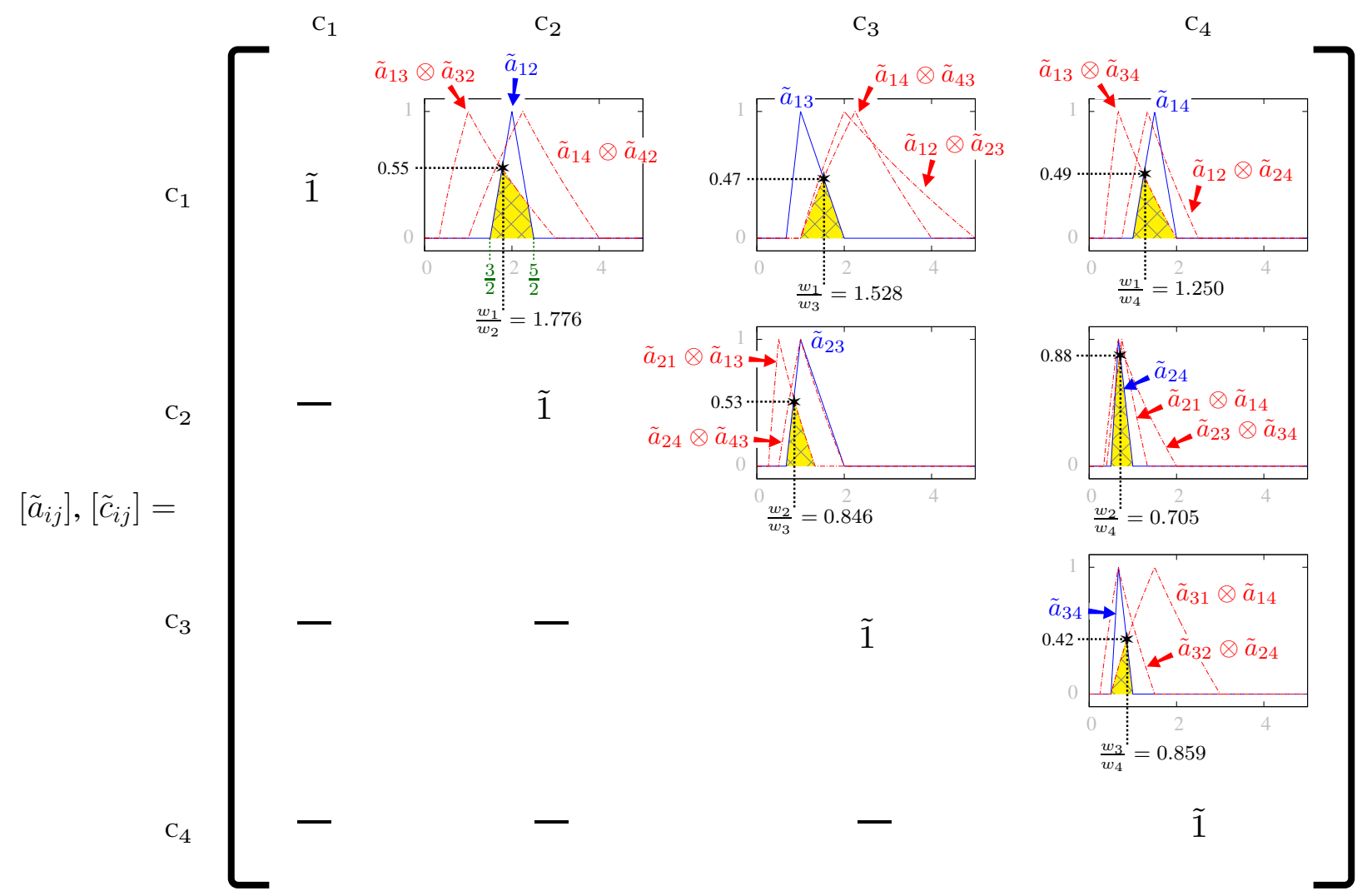

Figure 3: 4x4 FPCM specified in [49] by an expert for emergency response capacity assessment purposes

$\alpha=0$ when applying (7):

$$
\begin{aligned}
& =\max \left(\underline{a}_{12}^{\alpha} ;\left(\begin{array}{l}
\underline{a}_{13}^{\alpha} \times \underline{a}_{32}^{\alpha} \\
\underline{a}_{14}^{\alpha} \times \underline{a}_{42}^{\alpha}
\end{array}\right)\right) \leq \min \left(\bar{a}_{12}^{\alpha} ;\left(\begin{array}{c}
\bar{a}_{13}^{\alpha} \times \bar{a}_{32}^{\alpha} \\
\bar{a}_{14}^{\alpha} \times \bar{a}_{4}^{\alpha} 2
\end{array}\right)\right)=\max \left(\frac{3}{2} ;\left(\begin{array}{c}
2 / 3 \times 1 / 2 \\
1 \times 1
\end{array}\right)\right) \leq \min \left(\frac{5}{2} ;\left(\begin{array}{c}
2 \times 3 / 2 \\
2 \times 2
\end{array}\right)\right) \\
& =\frac{3}{2} \leq \frac{5}{2}
\end{aligned}
$$

The inequality is satisfied, and therefore $c_{12}^{0}$ is said to be consistent. Here, $\frac{3}{2}$ and $\frac{5}{2}$ respectively correspond to the lower and upper interval values of the "support" of $\tilde{c}_{12}\left(c f\right.$., the yellow meshed shape $\mathrm{C}_{1,2}$ in Figure 3 ). Now, by examining the support interval of all the yellow meshed shapes (i.e., $\forall i, j$ ), it can be concluded that Theorem 1 is satisfied for the whole FPCM.

By considering Theorem 2 (14), the degree of consistency can be computed. Firstly, all the $\tilde{c}_{i j}$ elements are computed, giving the yellow meshed shapes (the intersection between the direct and indirect expert knowledge). Secondly, $\alpha_{\mathrm{KCI}}$ is computed, which can graphically be described graphically as the minimal vertex/top value of the yellow meshed shapes (this value is represented through the $*$ symbol in Figure 3 ). By applying (5) along with the minimal vertex/top values, $\alpha_{\mathrm{KCI}}$ is determined in (24).

$$
\begin{aligned}
\alpha_{\mathrm{KCI}} & =\min \left(\sup \left(\tilde{c}_{12}\right), \sup \left(\tilde{c}_{13}\right), \ldots, \sup \left(\tilde{c}_{34}\right)\right) \\
& =\min (0.55,0.47,0.49,0.53,0.88,0.42) \\
& =0.42
\end{aligned}
$$

Here, $\tilde{A}$ gives a KCI-consistent of value 0.42 , bearing in mind that: "the higher $\alpha_{K C I}(\in[0 ; 1])$, the more axiomatically consistent the expert knowledge is, and the more satisfied they will be." Furthermore, this ensures that there 


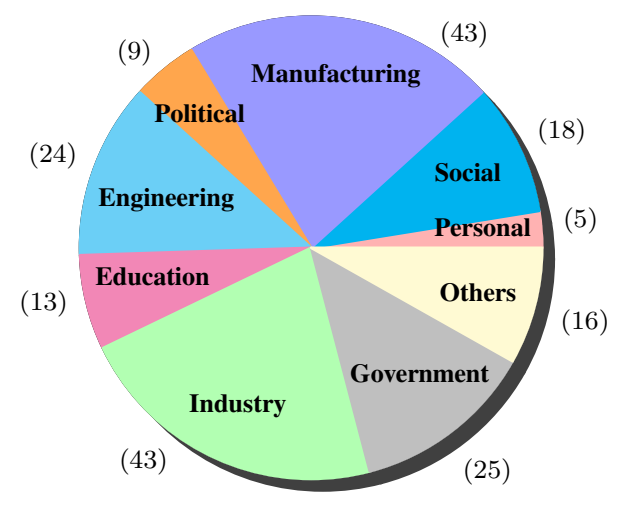

(a) Application area-specific distribution

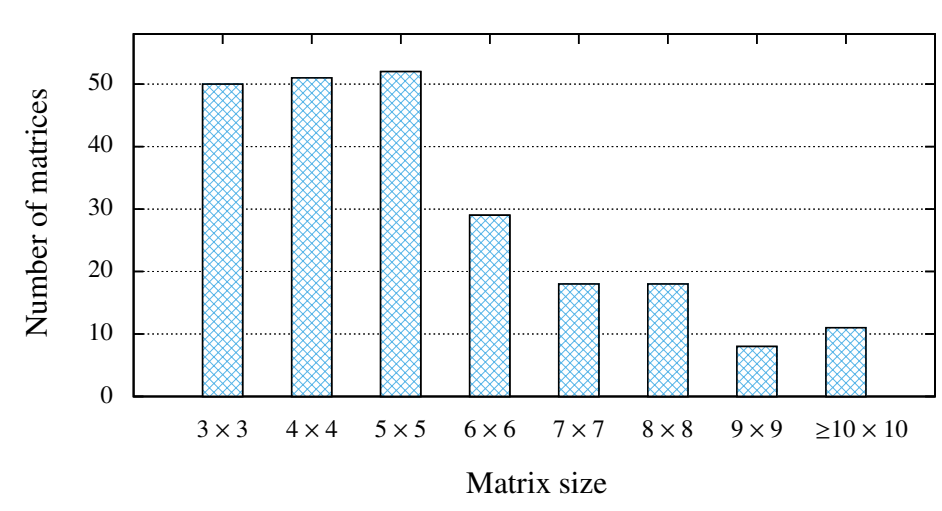

(b) Distribution of FPCMs according their size $n$

Figure 4: Distribution of the scientific papers - from which the FPCM $(s)$ were selected - arranged by application and matrix size

exists a consistent crisp matrix along with its eigenvector solution, which are derived from $\tilde{A}$ using (20), as given in (25) (eigenvector solution being denoted by EV). For such a matrix/eigenvector, the consistency ratio (CR) is equal to 0 , thus confirming that the crisp comparison matrix derived from $\tilde{c}_{i j}$ is fully consistent according to Saaty's definition.

$$
\begin{aligned}
& \begin{array}{lllll}
\mathrm{C} 1 & \mathrm{C} 2 & \mathrm{C} 3 & \mathrm{C} 4 & \mathrm{EV}
\end{array} \\
& \begin{array}{l}
\text { C1 } \\
\text { C2 } \\
\text { C3 } \\
\text { C4 } 4
\end{array}\left[\begin{array}{cccc}
1 & 1.776 & 1.528 & 1.250 \\
0,654 & 1 & 0.846 & 0.705 \\
0.800 & 1,418 & 1 & 0.859 \\
0.164 & 1
\end{array}\right]\left[\begin{array}{l}
0.332 \\
0.186 \\
0.220 \\
0.262
\end{array}\right]
\end{aligned}
$$

\subsection{KCI behavior analysis $\mathcal{E}$ parameterization}

To carry out a suitable analysis of the proposed KCI, we employed the FAHP testbed ${ }^{3}$, released in the recent stateof the-art survey presented in [5]. This testbed makes available one or more FPCM(s) from the corpus of reviewed papers available (190 research papers published between 2006 and 2016), giving a total of 237 matrices (set denoted by $\mathcal{F}$ ). Figures $4($ a) and 4(b) provide an overview of both (i) the application domain covered by the 190 papers, and (ii) the distribution of the 237 FPCMs according to their respective sizes ${ }^{4}$. To study KCI, equation (5) was implemented under in a MATLAB environment, where the membership functions $\tilde{a}_{i j}$ were discretized (discretization of the support of membership functions) in order to be able to compute $\alpha_{\mathrm{KCI}}$. We therefore propose to study the impact of such a discretization, both on the obtained $\alpha_{\mathrm{KCI}}$ score and on the time required to perform the calculation. Such analyses are respectively illustrated in Figure 5(a) and 5(b), where the $x$-axis corresponds to the set of discretization levels (a logarithmic scale is employed in Figure 5(a), with a linear one in Figure 5(b)).

In Figure 5(a), the $y$-axis corresponds to the average $\alpha_{\mathrm{KCI}}$ score obtained considering all matrices of size $3 \times 3,4 \times 4$, and so on. Two observations can be drawn: (i) The higher the discretization level is, the higher (or more precise) the $\alpha_{\mathrm{KCI}}$ scores, and (ii) the $\alpha_{\mathrm{KCI}}$ scores reach their maximum for a discretization level of 10000 (all curves being stable after this level). The second observation represents an important finding, because it enables us to identify the upper discretization index, namely the one ensuring that the algorithm has converged to its maximum possible precision.

The second analysis (see Figure 5(b)) provides an insight into the the average time required to compute $\alpha_{\mathrm{KCI}}$ considering all matrices of size $3 \times 3,4 \times 4$, and so on. Two observations can be drawn: (i) The time complexity for computing $\alpha_{\mathrm{KCI}}$ grows linearly, and (ii) the average time required to compute $\alpha_{\mathrm{KCI}}$ with a discretization level of 10000 is always smaller than $4 s$, as highlighted in the enlarged view provided in Figure 5(b).

\footnotetext{
${ }^{3}$ Testbed's URL: http://fahptestbed.jeremy-robert.fr

${ }^{4}$ FPCMs of size is greater than $10 \times 10-$ up to $20 \times 20$ - are summed over under the $\geq 10 \times 10 x$-label.
} 


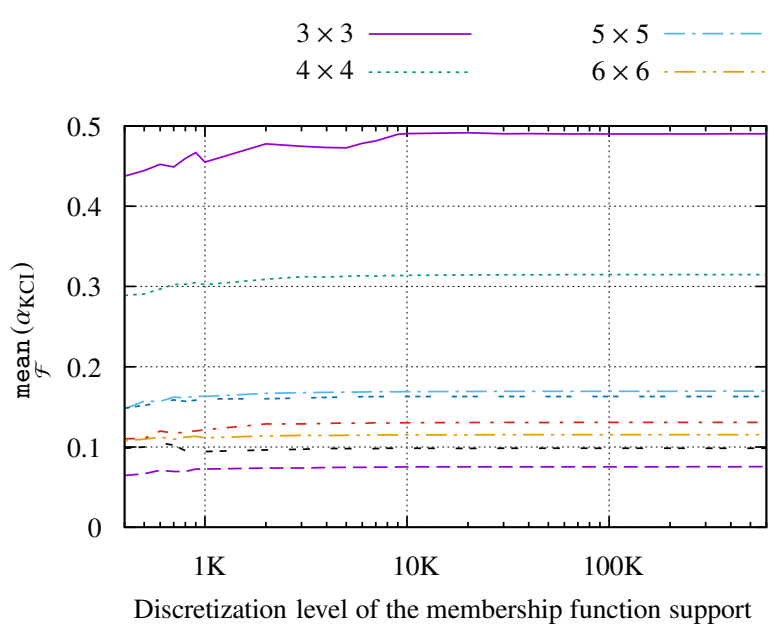

(a) Score obtained for $\alpha_{\mathrm{KCI}}$ according to different discretization levels

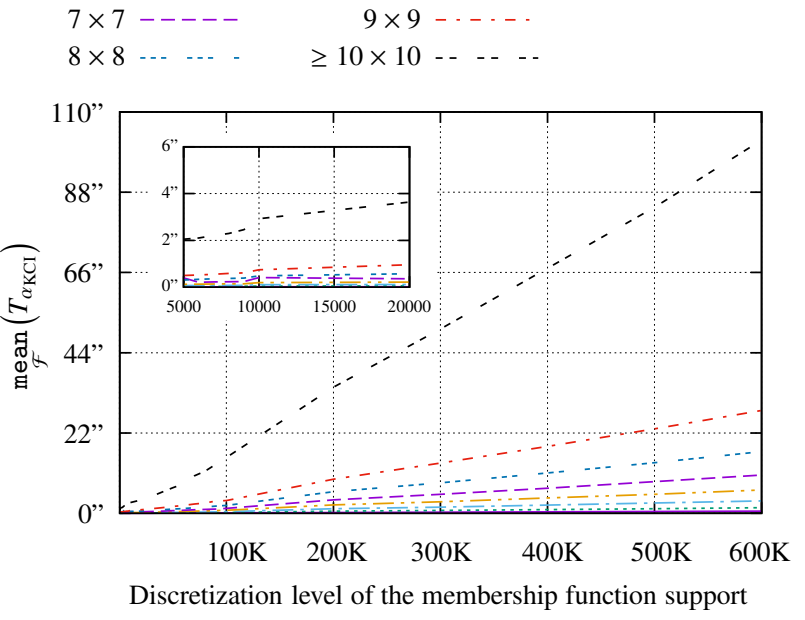

(b) Time required to compute $\alpha_{\mathrm{KCI}}$ according to different discretization levels

Figure 5: Impact analysis of the discretization on $\alpha_{\mathrm{KCI}}$ and the calculation time

Table 1: Experimental data and outcomes: Ohnishi's index vs. KCI

\begin{tabular}{lccccccccc} 
Size & $3 \times 3$ & $4 \times 4$ & $5 \times 5$ & $6 \times 6$ & $7 \times 7$ & $8 \times 8$ & $9 \times 9$ \\
\hline Score similarity & $100 \% \Delta^{1.7 \%}$ & $100 \% \Delta^{0.2 \%}$ & $100 \% \Delta^{0.2 \%}$ & $100 \% \Delta^{0.6 \%}$ & $100 \% \Delta^{0.1 \%}$ & $100 \% \Delta^{1.2 \%}$ & $100 \% \Delta^{4.3 \%}$ & $100 \% \Delta^{0.1 \%}$ \\
Consistent FPCMs & $88 \%$ & $65 \%$ & $43 \%$ & $39 \%$ & $28 \%$ & $22 \%$ & $25 \%$ & $20 \%$ \\
Time difference (s) & {$[0.8 ; 25.7]$} & {$[0.9 ; 10.5]$} & {$[1.0 ; 25.8]$} & {$[0.2 ; 29.8]$} & {$[2.1 ; 72.4]$} & {$[3.6 ; 312]$} & {$[15.6 ; 365.9]$} & {$[35 ; 7360]$} \\
\hline
\end{tabular}

Based on the above findings, it can be concluded that the best compromise for achieving the highest possible $\alpha_{\mathrm{KCI}}$ score in a reasonable computational time is given by a discretization level of 10000 . This discretization is therefore employed for the comparison study presented in the next section.

\subsection{Comparison study: KCI vs. Ohnishi}

In order to compare KCI with a state-of-the-art consistency index, the one introduced by Onhishi has been considered and implemented. Both $\alpha_{\mathrm{KCI}}$ and $\alpha_{\mathrm{Ohn}}$ are implemented, where criteria defined for the purposes of comparison are the "score similarity" between the two indexes and the "computation time" required by each. Table 1 provides an overview of the results/findings of our study. First, in terms of the score similarity it can be observed that both indexes have identical scores (in $100 \%$ of cases), with a maximum deviation of $4.3 \%$ (cf., $\Delta^{\%}$ in Table 1 ). This means that from a consistency standpoint $\alpha_{\mathrm{KCI}}$ and $\alpha_{\mathrm{Ohn}}$ perform on a similar level.Table 1 also presents the proportion of consistent FPCMs (of the 237 matrices) per size category ( $c f$., "Consistent FPCMs"). It can be observed that $88 \%$ of the $3 \times 3$ FPCMs are consistent $\left(\alpha_{\mathrm{Ohn}}=\alpha_{\mathrm{KCI}}>0\right)$, while this trend decreases along with the increase in the FPCMs' size. For example, only 20 to $28 \%$ of large FPCMs $(7 \times 7$ to $\geq 10 \times 10)$ are consistent, compared with 65 to $88 \%$ for small size matrices (e.g., $3 \times 3$ and $4 \times 4$ ). This appears to be a logical finding, because the human brain experiences more difficulty when comparing an increased number of criteria in a pairwise manner.

Figure 6(a) provides a more in-depth overview of the scores obtained for the set of consistent FPCMs (e.g., regarding the $65 \%$ of consistent $4 \times 4$ FPCMs). This graph highlights the min, avg and max consistency scores of these sets of FPCMs per size category. Interestingly, the average consistency scores remain between 0.3 and 0.6 (see * in Figure 6(a)), and slowly decrease with the increase of the matrix size, except for the $8 \times 8$ and $\geq 10 \times 10$ FPCMs. However, this could be partly explained by the fact that the number of consistent FPCMs in the upper size range is limited. Furthermore, the total number of FPCMs from the outset in the upper size range is smaller compared with in the lower range ( $c f$., Figure 4(b)). For example, the $4 \times 4$ size category consists of 51 FPCMs, compared with 18 FPCMs for the $8 \times 8$ size category. In addition, $65 \%$ of the 51 FPCMs are consistent (i.e., 33\% FPCMs) compared with $22 \%$ in the latter case (i.e., only 3 FPCMs). These factors are likely to have an impact on the relevance of the 


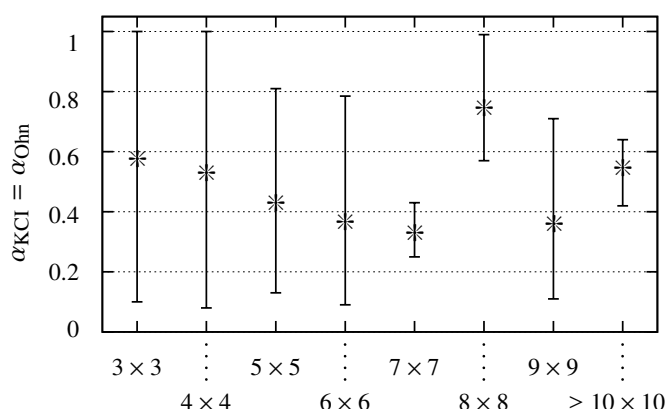

FPCM size

(a) Consistency scores obtained by $\alpha_{\mathrm{KCI}}$ and $\alpha_{\mathrm{Ohn}}$

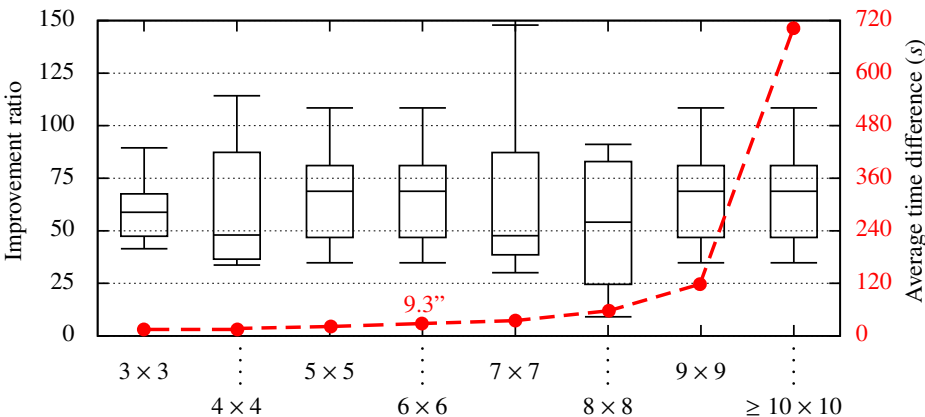

FPCM size

(b) Comparison of the required"computation time"

Figure 6: Comparison of KCI vs. Ohnishi's index" from an efficiency and computation time viewpoints

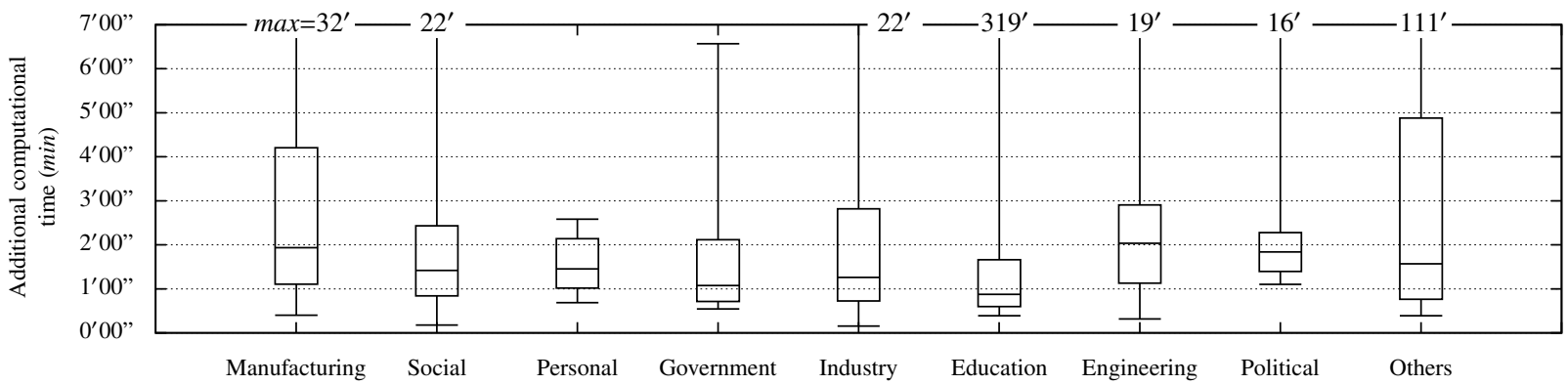

Figure 7: Estimated additional time required by experts to perform all pairwise comparisons (i.e., all FPCMs in an article) for each study/paper

min, avg and max consistency scores in the upper size range.

We now consider the computation times required by $\alpha_{\mathrm{KCI}}$ and $\alpha_{\mathrm{Ohn}}$. Table 1 provides an overview of the minimum and maximum differences between the times required by $\alpha_{\mathrm{Ohn}}$ and $\alpha_{\mathrm{KCI}}$ (i.e., $\left[\min \left(T_{\alpha_{\mathrm{Ohn}}}-T_{\alpha_{\mathrm{KCI}}}\right) ; \max \left(T_{\alpha_{\mathrm{Ohn}}}-T_{\alpha_{\mathrm{KCI}}}\right)\right]$ ). For example, for the $3 \times 3$ category, the values $0.8 \mathrm{~s}$ and $25.7 \mathrm{~s}$ respectively indicate that among the 50 FPCMs that compose this category, Ohnishi's index requires in the best case $0.8 \mathrm{~s}$ more than $\mathrm{KCI}$ to compute in the best case, and $25.7 \mathrm{~s}$ in the worst case. Figure 6(b) provides a more in-depth overview of the computation times and time difference, by introducing the following two indicators:

- Improvement ratio, $\left[T_{\alpha_{\mathrm{Ohn}}} / T_{\alpha_{\mathrm{KCI}}}\right]$ : the higher the ratio, the more efficiently $\alpha_{\mathrm{KCI}}$ performs compared to $\alpha_{\mathrm{Ohn}}$;

- Average time difference, avg $\left(\left[T_{\alpha_{\mathrm{Ohn}}}-T_{\alpha_{\mathrm{KCI}}}\right]\right)$ : The average of the time differences (per size category). The higher the average time difference, the more meaningful the improvement ratio is, e.g. an improvement ratio of 40 is considerably less meaningful on a scale of milliseconds (if $T_{\alpha_{\mathrm{Ohn}}}=1 \mathrm{~ms}$, then $T_{\alpha_{\mathrm{KCI}}}=\frac{1 \mathrm{~ms}}{20}=0.05 \mathrm{~ms}$ ) than with seconds or minutes (if $T_{\alpha_{\mathrm{Ohn}}}=5 \mathrm{~min}$, then $T_{\alpha_{\mathrm{KCI}}}=\frac{5 \min }{20}=15 \mathrm{~s}$ ).

The improvement ratio boxplots presented in Figure 6(b) show that $\alpha_{\mathrm{KCI}}$ always performs faster than $\alpha_{\mathrm{Ohn}}$, because in $50 \%$ of cases, $\alpha_{\mathrm{KCI}}$ performs 25 to 80 times faster than $\alpha_{\mathrm{Ohn}}$, and up to between 80 and 150 times in $25 \%$ of other cases (i.e., values above the $3^{r d}$ quartile). When inferring these results with the average time difference ( $c f$., the red curve in Figure 6(b)), it can be observed that the computation time increases (following an exponential curve) with the increase in the FPCM size, ranging from a few seconds/minutes when dealing with $\leq 7 \times 7$ FPCMs to approximatively 15 min (up to several hours) when dealing with $\geq 10 \times 10$ FPCMs. The reason for this is that $\alpha_{\mathrm{KCI}}$ does not require any optimization stage, unlike $\alpha_{\mathrm{Ohn}}$ as was discussed in Section 2.3. 
It should be noted that in a typical FAHP study, decision maker(s) deal with more than one single FPCM, depending on the number of criteria levels and alternatives. Therefore, this can therefore become a time-consuming task, particularly owing to the re-examination that is required when consistency is not satisfied [3, 50, 51]. In the following, we attempt to provide a rough estimate of how time-consuming this could be. For this, we examined into all research articles composing the testbed (i.e., 190 articles), and identified the total number of FPCMs carried out by decision makers considering their MCDM problem. Given this number, we then estimated the additional computational time that would be necessary - for decision makers - to handle all FPCMs in the case that they use $\alpha_{\mathrm{Ohn}}$ rather than $\alpha_{\mathrm{Ohn}}$. These times are estimated based on the average time difference identified in Figure 6(b) (this is why we refer to a "rough estimate"). Let us consider the MCDM problem given in [52], where nine FPCMs of size $5 \times 5$ are performed by a decision maker, thus leading to the following estimate: 9 FPCMs $\times 9.3$ " $=84$ " (9.3" being emphasized in Figure 6(b)). Figure 7 provides a global overview of the additional times that have been estimated on the basis of the 190 papers. These have been grouped on the $x$-axis based on the application domain addressed by each paper ( $c f$., Figure 7). Although the domain is not of prime importance, this enables us to observe that (i) times follow the same distribution between 1 and 3 minutes for most domains), (ii) it can become time consuming to deal with consistency in all FPCMs when considering the MCDM problem as a whole (up to between one half and several hours for some MCDM studies), which can become a problem for some decision makers when judgements require re-examination. Although the vast majority of MCDM problems are tackled in a non-time-sensitive fashion, some studies employ MCDM techniques to deal with real-time decisions (see, e.g., [53], where the authors deal with an open data portal ranking over time for $e$-government purposes), which would therefore be impacted by such computational time.

\subsection{Impact on FPCM consistency of employing Chang's extent analysis method}

The recent state-of-the-art survey of FAHP applications in [5] presented evidence that the Chang's extent analysis method [40] is presently one of the most widely techniques today (109 out of the 190 reviewed papers), despite many criticisms. Indeed, a significant number of research papers have demonstrated that this method suffers from theoretical pitfalls, particularly for deriving the true weights from FPCMs. Given this fact, it is worth analyzing the extent to which the corpus of studies employing this method may suffer from inconsistent pairwise comparison matrices on the basis of the crisp matrix derived from $\alpha_{\mathrm{Ohn}}$ and $\alpha_{\mathrm{KCI}}(c f$., Section 3.3 and Eq. 25).

Figure 8(a) provides a first overview of the percentages of resulting consistent and inconsistent pairwise comparison matrices. It can be noted that, over a total of 237 FPCMs, 54\% of the matrices turned out to be consistent based on Saaty's definition (i.e., CR< 10\%), and $46 \%$ were inconsistent (i.e., CR $>10 \%$ ). Now, examining the proportion of FPCMs that were deemed likely ${ }^{5}$ to be consistent, it can be observed that $60 \%$ of the inconsistent matrices originate from studies employing the Chang's extent analysis method. This is an interesting finding, because to some extent it confirms that the theoretical pitfalls of the extent analysis lead to a larger proportion of pairwise comparison matrices being inconsistent.

Figure 8(b) provides a more in-depth overview of the $46 \%$ of inconsistent pairwise comparison matrices, by plotting the percentage of inconsistent matrices per FPCM size category ( $x$-axis), while highlighting the proportion of matrices originating from studies employing Chang's extent analysis. This histogram provides a graphic display of the statement presented in Section 4.3, namely that "the higher the number of criteria to be compared in a pairwise manner, the more difficult it becomes for the human brain," which is even more true when incorporating uncertainty into the decision-making. Finally, it can be noted that the proportion of inconsistent matrices whose approach leverages on the Chang's extent analysis, is particularly high for FPCMs of size $\geq 7 \times 7$, although it is also around $50 \%$ for the lower sizes.

\section{Conclusion and Research Implications, Limitations and Perspectives}

\subsection{Conclusion}

Fuzzy logic has been introduced in AHP as a method of copying with the uncertainty and vagueness arising from pairwise comparisons carried out by decision makers (i.e., when comparing items using FPCMs). While such

\footnotetext{
5"Likely" because not all MCDM studies develop, or at least present, a consistency analysis of their FPCMs.
} 


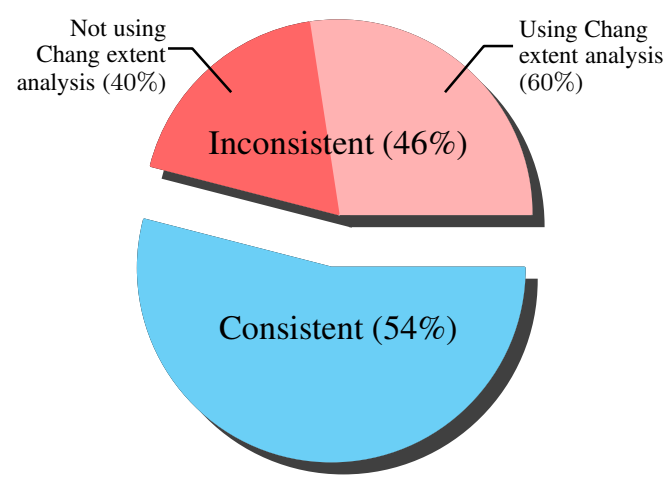

(a) Application area-specific distribution

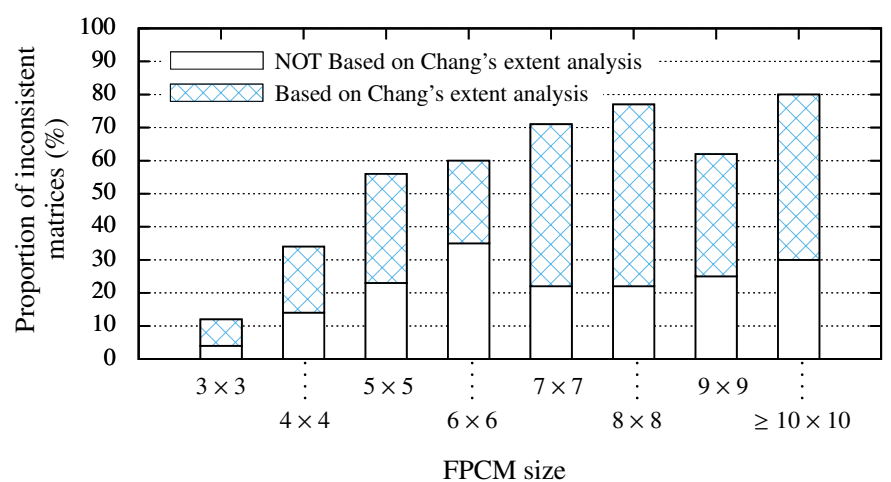

(b) Distribution of FPCMs according their size

Figure 8: Consistency analysis of the 237 FPCMs (from the FAHP testbed) with an emphasis on the use of the Chang's extent analysis

an idea seems wise, its applications give rise to several theoretical pitfalls concerning the axiomatic foundation of introducing fuzzy sets in AHP. When dealing with FPCMs, and even with crisp pairwise comparison matrices, one of the main concerns related to the consistency of the matrices. Indeed, it is obvious that a consistent knowledge is a prerequisite for a matrix to be effective and for its computation in the in later stage of every method. While for AHP Saaty introduced a method of evaluating the consistency of a crisp pairwise comparison matrix, several consistency indexes have been introduced for dealing with FPCMs. In this paper, consistency indexes introduced over the past four decades have been reviewed and discussed. Despite their various pros and cons, many of the criticisms are directed towards their failure to be "axiomatically" grounded. Based on the reviewed papers, the consistency index introduced by Ohnishi et al. [45] appears to be axiomatically well founded, viewing the FPCM as representing knowledge about preference relations.

A new consistency index, called the knowledge-based consistency Index (KCI) is proposed in the present paper, which helps decision makers to measure the consistency of the different items of knowledge expressed in any triangular FPCM, while being able to derive a crisp solution vector that is always perfectly consistent in the sense of Saaty. Like Ohnishi's index, KCI does not - theoretically speaking - correspond to Saaty's index, but it can be seen as a natural substitute for evaluating and measuring the degree of consistency in any triangular FPCM. KCI is also axiomatically well founded, but unlike Ohnishi's index it does not involve any optimization stage for the crisp solution vector derivation process, thus contributing to reducing the computation time required to reach the same result. This effect has been demonstrated through a set of experiments, the results of which show that computation time can vary greatly between the two methods (up to several hours in some cases). Thus, our method is then thought to be simpler and faster to employ than Ohnishi's approach, or indeed any other index that would involve optimization stages. All results and datasets of our experiments have been made fully and freely available at http://fahptestbed.jeremy-robert.fr.

\subsection{Research implications, limitations, and perspectives}

The research presented in this paper deals with the consistency problem in MCDM problems under preference relations, which turns to be an important issue in intelligent decision making systems. It should be noted that the contributions of this research do not represent the MCDM level, but rather the FPCM level. To put it another way, the goal is not to select the most appropriate MCDM technique considering a given MCDM problem (e.g., AHP, VIKOR, or TOPSIS), but rather to propose an approach that helps decision makers who have decided to use FAHP to better tackle (in)consistency in fuzzy pairwise comparison matrices (FPCMs).

The KCI index is currently only specified and employed with triangular fuzzy sets. However, one may wonder whether KCI could be generalized to FPCM dealing with other types of fuzzy sets (e.g., trapezoidal fuzzy sets), and therefore applied in any problem concerning incomplete and/or missing preferences, such as those presented in $[54,55,56]$. Such a generalization could be achieved, although this would require some customization to tackle. For example, the kernel of the resulting $\tilde{c}_{i j}$ factors could be an interval rather than a single value, thus implying to re-think/customize step 4 of Algorithm 1 (as the intersections would result in a set of maximum values for $x$. 
Another important research direction involves studying the case that decision makers should modify their pairwise matrix depending on the KCI score (this corresponds to the loop "if KCI too low" in Figure 2). It is indeed important to study and specify which minimal threshold(s) would require modifying the FPCM, but also to propose an approach to guide the decision maker in such a modification process. For example, one may imagine an algorithm that would measure how widely separated the direct and indirect knowledge expressed by the expert is, and based on this measure, one or more pairwise comparison modifications could be proposed to the decision maker. To this end, it could be worth exploring the possibility of coupling KCI with other indices such as the <delta $>$ and < sigma $>$ fuzzy parameters [47], as these are designed to minimize the number of modified elements, while maximizing the similarity of the modified matrix with the original one. Considering the FPCM given in Figure 3, the algorithm could, for example, suggest modifying $\tilde{a}_{13}$ in order to drag the direct knowledge (i.e., the solid blue membership function) to the right side (i.e., towards the indirect knowledge, corresponding to the dashed red membership functions), even though the overall impact should be carefully studied before suggesting such a modification.

\section{Acknowledgment}

We wish express our gratitude to the experts who participated in peer-review process. This research is funded by the EU's H2020 Programme (grant 688203), as well as the FEDER financial support from the Project TIN201675850-P..

\section{References}

[1] L. Simpson, Do decision makers know what they prefer?: MAVT and ELECTRE II, Journal of the Operational Research Society 47 (7) (1996) 919-929.

[2] C. L. Hwang, K. Yoon, Multiple Attribute Decision-Making Methods and Applications, Springer Verlag, Berlin, Heidelberg, New York, 1981.

[3] T. L. Saaty, The Analytic Hierarchy Process, New York: McGraw-Hill, 1980.

[4] O. Vaidya, S. Kumar, Analytic hierarchy process: An overview of applications, European Journal of operational research 169 (1) (2006) $1-29$.

[5] S. Kubler, J. Robert, W. Derigent, A. Voisin, Y. Le Traon, A state-of the-art survey \& testbed of Fuzzy AHP (FAHP) applications, Expert Systems with Applications 65 (2016) 398-422.

[6] R. Ureña, F. Chiclana, J. A. Morente-Molinera, E. Herrera-Viedma, Managing incomplete preference relations in decision making: a review and future trends, Information Sciences 302 (2015) 14-32.

[7] R. Urena, F. Chiclana, E. Herrera-Viedma, Consistency based completion approaches of incomplete preference relations in uncertain decision contexts, in: IEEE International Conference on Fuzzy Systems (FUZZ-IEEE), 1-6, 2015.

[8] M. Brunelli, M. Fedrizzi, Boundary properties of the inconsistency of pairwise comparisons in group decisions, European Journal of Operational Research 240 (3) (2015) 765-773.

[9] P. Grošelj, L. Z. Stirn, Acceptable consistency of aggregated comparison matrices in analytic hierarchy process, European Journal of Operational Research 223 (2) (2012) 417-420.

[10] F. Liu, W.-G. Zhang, Z.-X. Wang, A goal programming model for incomplete interval multiplicative preference relations and its application in group decision-making, European Journal of Operational Research 218 (3) (2012) 747-754.

[11] D. J. Weiss, J. Shanteau, The vice of consensus and the virtue of consistency, Psychological investigations of competent decision making (2004) 226-240.

[12] C. Kahraman, Fuzzy multi-criteria decision making: theory and applications with recent developments, vol. 16, Springer Science \& Business Media, 2008

[13] L. Zadeh, Fuzzy sets, Information and Control 8 (1965) 338-353.

[14] P. van Laarhoven, W. Pedrycz, A fuzzy extension of Saaty's priority theory, Fuzzy Sets and Systems 11 (1) (1983) $199-227$.

[15] J. Buckley, Fuzzy hierarchical analysis, Fuzzy Sets and Systems 17 (1985) 233-247.

[16] A. A. Salo, On fuzzy ratio comparisons in hierarchical decision models, Fuzzy Sets and Systems 84 (1) (1996) $21-32$.

[17] Y.-M. Wang, K.-S. Chin, A linear programming approximation to the eigenvector method in the analytic hierarchy process, Information Sciences 181 (23) (2011) 5240-5248.

[18] J. Chu, X. Liu, Z. Gong, Two decision making models based on newly defined additively consistent intuitionistic preference relation, in: IEEE International Conference on Fuzzy Systems (FUZZ-IEEE), 1-8, 2015.

[19] E. Herrera-Viedma, F. Chiclana, F. Herrera, S. Alonso, Group decision-making model with incomplete fuzzy preference relations based on additive consistency, IEEE Transactions on Systems, Man, and Cybernetics, Part B (Cybernetics) 37 (1) (2007) 176-189.

[20] G. Crawford, C. Williams, A note on the analysis of subjective judgment matrices, Journal of mathematical psychology 29 (4) (1985) 387405.

[21] J. Ramík, P. Korviny, Inconsistency of pair-wise comparison matrix with fuzzy elements based on geometric mean, Fuzzy Sets and Systems 161 (11) (2010) 1604-1613.

[22] D. Dubois, The role of fuzzy sets in decision sciences: Old techniques and new directions, Fuzzy Sets and Systems 184 (1) (2011) 3-28.

[23] F. Meng, X. Chen, A New Method for Triangular Fuzzy Compare Wise Judgment Matrix Process Based on Consistency Analysis, International Journal of Fuzzy Systems (2016) 1-20. 
[24] Y.-M. Wang, Y. Luo, Z. Hua, On the extent analysis method for fuzzy AHP and its applications, European Journal of Operational Research 186 (2) (2008) 735-747.

[25] Y.-M. Wang, K.-S. Chin, Fuzzy analytic hierarchy process: A logarithmic fuzzy preference programming methodology, International Journal of Approximate Reasoning 52 (4) (2011) 541-553.

[26] K. Zhü, Fuzzy analytic hierarchy process: Fallacy of the popular methods, European Journal of Operational Research 236 (1) (2014) $209-217$.

[27] S. Kubler, W. Derigent, A. Voisin, J. Robert, Y. Le Traon, Knowledge-based Consistency Index for Fuzzy Pairwise Comparison Matrices, in: IEEE International Conference on Fuzzy Systems, 1-7, 2017.

[28] T. L. Saaty, A scaling method for priorities in hierarchical structures, Journal of mathematical psychology 15 (3) (1977) $234-281$.

[29] F. A. Lootsma, Performance evaluation of non-linear optimization methods via multi-criteria decision analysis and via linear model analysis, Academic Press, London, 419-453, 1981.

[30] C. G. E. Boender, J. G. De Graan, F. A. Lootsma, Multi-criteria decision analysis with fuzzy pairwise comparisons, Fuzzy sets and Systems 29 (2) (1989) 133-143.

[31] O. Gogus, T. O. Boucher, Strong transitivity, rationality and weak monotonicity in fuzzy pairwise comparisons, Fuzzy Sets and Systems 94 (1) (1998) 133-144.

[32] Y.-M. Wang, T. Elhag, Z. Hua, A modified fuzzy logarithmic least squares method for fuzzy analytic hierarchy process, Fuzzy Sets and Systems 157 (23) (2006) 3055-3071.

[33] J. Aguaron, J. M. Moreno-Jiménez, The geometric consistency index: Approximated thresholds, European Journal of Operational Research 147 (1) (2003) 137-145.

[34] E. Bulut, O. Duru, T. Keçeci, S. Yoshida, Use of consistency index, expert prioritization and direct numerical inputs for generic fuzzy-AHP modeling: A process model for shipping asset management, Expert Systems with Applications 39 (2) (2012) 1911-1923.

[35] E. Dopazo, K. Lui, S. Chouinard, J. Guisse, A parametric model for determining consensus priority vectors from fuzzy comparison matrices, Fuzzy Sets and Systems 246 (2014) 49-61.

[36] Y.-M. Wang, J.-B. Yang, D.-L. Xu, K.-S. Chin, On the centroids of fuzzy numbers, Fuzzy sets and systems 157 (7) (2006) $919-926$.

[37] J. Ramík, P. Korviny, Measuring Inconsistency of Pair-wise Comparison Matrix with Fuzzy Elements, International Journal of Operations Research 10 (2) (2013) 100-108.

[38] R. Csutora, J. J. Buckley, Fuzzy hierarchical analysis: the Lambda-Max method, Fuzzy sets and Systems 120 (2) (2001) 181-195.

[39] Y.-M. Wang, K.-S. Chin, An eigenvector method for generating normalized interval and fuzzy weights, Applied mathematics and computation 181 (2) (2006) 1257-1275.

[40] D.-Y. Chang, Applications of the extent analysis method on fuzzy AHP, European Journal of Operational Research 95 (3) (1996) $649-655$.

[41] A. Arbel, Approximate articulation of preference and priority derivation, European Journal of Operational Research 43 (3) (1989) 317-326.

[42] L. Leung, D. Cao, On consistency and ranking of alternatives in fuzzy AHP, European Journal of Operational Research 124 (1) (2000) $102-113$.

[43] L. Mikhailov, Deriving priorities from fuzzy pairwise comparison judgements, Fuzzy Sets and Systems 134 (3) (2003) $365-385$.

[44] H.-J. Zimmermann, Fuzzy set theory-and its applications, Springer Science \& Business Media, 2011.

[45] S.-I. Ohnishi, D. Dubois, H. Prade, T. Yamanoi, A fuzzy constraint-based approach to the analytic hierarchy process, in: Uncertainty and intelligent information systems, World Scientific, 217-228, 2008

[46] T. L. Saaty, L. T. Tran, On the invalidity of fuzzifying numerical judgments in the Analytic Hierarchy Process, Mathematical and Computer Modelling 46 (7-8) (2007) 962-975.

[47] H. Zhang, A. Sekhari, Y. Ouzrout, A. Bouras, Deriving consistent pairwise comparison matrices in decision making methodologies based on linear programming method, Journal of Intelligent \& Fuzzy Systems 27 (4) (2014) 1977-1989.

[48] Y.-M. Wang, J.-B. Yang, D.-L. Xu, Interval weight generation approaches based on consistency test and interval comparison matrices, Applied Mathematics and Computation 167 (1) (2005) 252-273.

[49] Y. Ju, A. Wang, X. Liu, Evaluating emergency response capacity by fuzzy AHP and 2-tuple fuzzy linguistic approach, Expert Systems with Applications 39 (8) (2012) 6972-6981.

[50] S. Karapetrovic, E. S. Rosenbloom, A quality control approach to consistency paradoxes in AHP, European Journal of Operational Research 119 (3) (1999) 704-718.

[51] C. A. B. e Costa, J.-C. Vansnick, A critical analysis of the eigenvalue method used to derive priorities in AHP, European Journal of Operational Research 187 (3) (2008) 1422-1428.

[52] P. Mohammady, A. Amid, Integrated Fuzzy AHP and Fuzzy VIKOR Model for Supplier Selection in an Agile and Modular Virtual Enterprise, Fuzzy Information and Engineering 3 (4) (2011) 411-431.

[53] S. Kubler, J. Robert, S. Neumaier, J. Umbrich, Y. Le Traon, Comparison of Metadata quality in Open Data portals using the Analytic Hierarchy Process, Government Information Quarterly .

[54] S. Alonso, F. Chiclana, F. Herrera, E. Herrera-Viedma, J. Alcalá-Fdez, C. Porcel, A consistency-based procedure to estimate missing pairwise preference values, International Journal of Intelligent Systems 23 (2) (2008) 155-175.

[55] F. Chiclana, E. Herrera-Viedma, S. Alonso, A note on two methods for estimating missing pairwise preference values, IEEE Transactions on Systems, Man, and Cybernetics, Part B (Cybernetics) 39 (6) (2009) 1628-1633.

[56] S. Alonso, E. Herrera-Viedma, F. Chiclana, F. Herrera, Individual and social strategies to deal with ignorance situations in multi-person decision making, International Journal of Information Technology \& Decision Making 8 (02) (2009) 313-333. 


\section{Appendix A. Acronyms and Variables}

Table A.2: Acronyms \& Variables used in this article

\begin{tabular}{|c|c|c|}
\hline \multirow{11}{*}{ Acronyms } & MCDM, MADM, MODM & Multiple $\{$ Criteria, Attribute, Objective $\}$ Decision-Making \\
\hline & SWOT & Strenghs, Weaknesses, Opportunities, Threats \\
\hline & (F)AHP & (Fuzzy) Analytic Hierarchy Process \\
\hline & FPCM & Fuzzy Pairwise Comparison Matrices \\
\hline & FFR & Fuzzy Feasible Region \\
\hline & FFLS & Fuzzy Logarithmic Least Square \\
\hline & $\mathrm{DF}$ & Direct Fuzzification \\
\hline & $\mathrm{KCI}$ & Knowledge-based Consistency Index \\
\hline & LLSM & Logarithmic Least Square Methods \\
\hline & $\mathrm{CCI}$ & Centric Consistency Index \\
\hline & $\mathrm{CR}, \mathrm{CI}, \mathrm{EV}$ & Consistency Ratio, Consistency Index, EigenVector \\
\hline \multirow{12}{*}{ Variables } & $\tilde{A}=\left[\tilde{a}_{i j}\right]$ & $\begin{array}{l}\text { A fuzzy pairwise comparison number, where } \tilde{a}_{i j} \text { is supposed to reflect, with some level of } \\
\text { imprecision, the factor by which the } i^{t h} \text { item is preferred to the } j^{t h} \text {. }\end{array}$ \\
\hline & $A^{\alpha}=\left[\tilde{a}_{i j}^{\alpha}\right]$ & The $\alpha$-cut matrix (also called alpha-matrix) of $\tilde{A}$. \\
\hline & $\operatorname{dim}(\tilde{A})$ & Size of $\tilde{A}$ \\
\hline & $\tilde{W}=\left(\tilde{w}_{1}, \ldots, \tilde{w}_{n}\right)=\left(\left(w_{1}^{L}, w_{1}^{M}, w_{1}^{U}\right)\right)$ & Normalized triangular fuzzy weight vector \\
\hline & $\alpha_{\mathrm{KCI}}, \alpha_{\mathrm{Ohn}}$ & Consistency indexes developed in this paper $\left(\alpha_{\mathrm{KCI}}\right)$ and by Ohnishi et al. [45] \\
\hline & $\underline{a}_{i j}^{\alpha}, \bar{a}_{i j}^{\alpha}$ & Respectively the lower and upper boundaries of the $\alpha$-cut interval \\
\hline & $c_{i j}, \tilde{c}_{i j}$ & $\begin{array}{l}\text { Fuzzy number that synthetizes the decision maker's knowledge issued from the direct and } \\
\text { indirect knowledges between the } t^{t h} \text { and } j^{t h} \text { elements. }\end{array}$ \\
\hline & $\tilde{C}^{\mathrm{KCI}}$ & The crisp consistent matrix derived from $\tilde{A}$ (using $\mathrm{KCI}$ ) \\
\hline & $\mathcal{H}(\tilde{A})$ & $\begin{array}{l}\mathcal{H} \text { is the height of the fuzzy set, or to put it another way the largest membership degree of } \\
\text { fuzzy set } \mathcal{H}(\tilde{A})=\sup _{x}\left(\mu_{\tilde{A}}(x)\right)\end{array}$ \\
\hline & $T_{\alpha_{\mathrm{Ohn}}}, T_{\alpha_{\mathrm{KCI}}}$ & Computational time required to compute Ohnishi's index $\left(\alpha_{\mathrm{Ohn}}\right)$ and $\mathrm{KCI}\left(\alpha_{\mathrm{KCI}}\right)$ \\
\hline & & Set of FPCM used for experimental purposes ( 237 matrices in total) \\
\hline & $\Delta^{\%}$ & Maximum deviation between Ohnishi's and KCI's index scores \\
\hline
\end{tabular}

\section{Appendix B. Consistency indexes introduced in the literature to deal with FPCMs}


Table B.3: FFLS consistency indexes

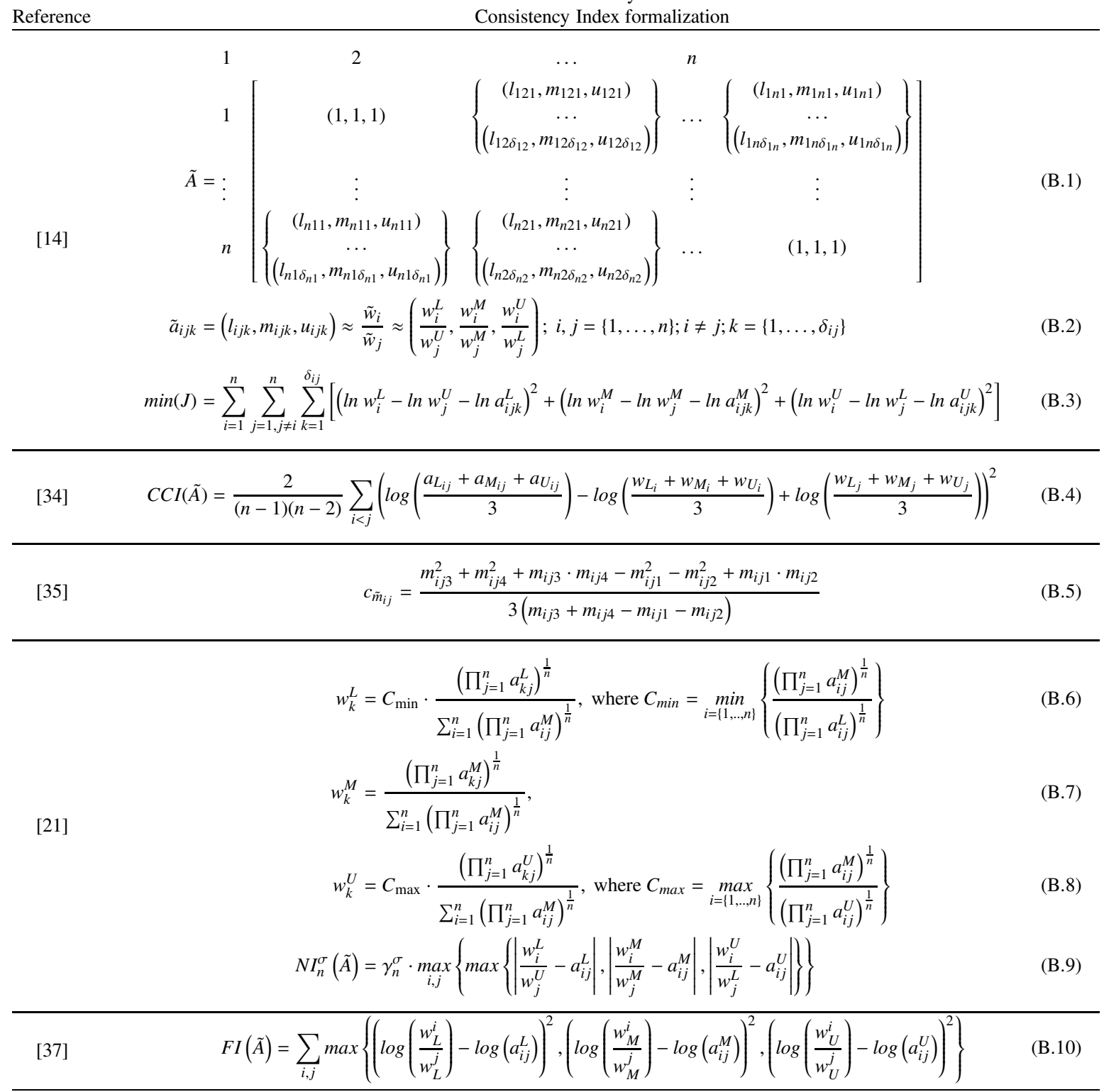


Table B.4: FFRM consistency indexes Consistency Index formalization

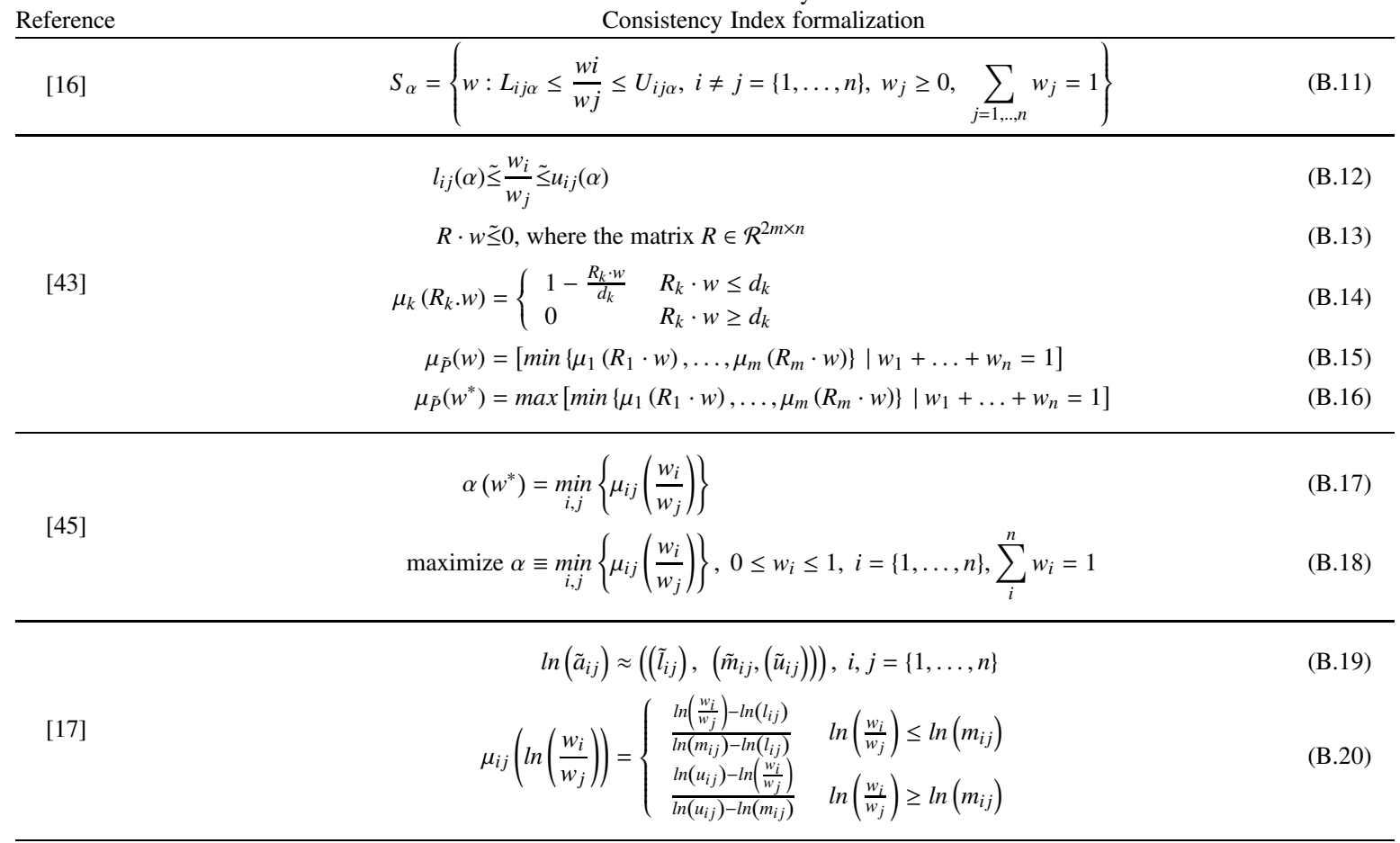

